Article

\title{
A CuAAC-Hydrazone-CuAAC Trifunctional Scaffold for the Solid-Phase Synthesis of Trimodal Compounds: Possibilities and Limitations
}

\author{
Benjamin Fabre, Jan Pícha, Václav Vaněk, Miloš Buděšínský and Jiří Jiráček * \\ Institute of Organic Chemistry and Biochemistry, Academy of Sciences of the Czech Republic, \\ v.v.i., Flemingovo n. 2, 16610 Praha 6, Czech Republic; E-Mails: fabre@uochb.cas.cz (B.F.); \\ picha@uochb.cas.cz (J.P.); vasek@uochb.cas.cz (V.V.); budesinsky@uochb.cas.cz (M.B.) \\ * Author to whom correspondence should be addressed; E-Mail: jiracek@uochb.cas.cz; \\ Tel.: +420-220-183-441.
}

Academic Editor: Fernando Albericio

Received: 28 August 2015 / Accepted: 16 October 2015 / Published: 23 October 2015

\begin{abstract}
We present a trifunctional scaffold designed for the solid-phase synthesis of trimodal compounds. This scaffold holds two alkyne arms in a free and TIPS-protected form for consecutive CuAAC (copper(I)-catalyzed azide-alkyne cycloaddition), one Fmoc-protected hydrazide arm for reaction with aldehydes, and one carboxylic acid arm with $\mathrm{CF}_{2}$ groups for attachment to the resin and ${ }^{19} \mathrm{~F}-\mathrm{NMR}$ quantification. This scaffold was attached to a resin and derivatized with model azides and aliphatic, electron-rich or electron-poor aromatic aldehydes. We identified several limitations of the scaffold caused by the instability of hydrazones in acidic conditions, in the presence of copper during CuAAC, and when copper accumulated in the resin. We successfully overcame these drawbacks by optimizing synthetic conditions for the derivatization of the scaffold with aromatic aldehydes. Overall, the new trifunctional scaffold combines CuAAC and hydrazone chemistries, offering a broader chemical space for the development of bioactive compounds.
\end{abstract}

Keywords: click chemistry; multifunctional scaffold; solid-phase synthesis; protein mimics; copper; hydrazone; hydrazide 


\section{Introduction}

Multifunctional scaffolds are precious tools used in chemical biology [1] and polymer science [2]. Such scaffolds bear two or more arms, each with orthogonal chemical properties. This allows for the construction of complex multimodal structures by simply "plugging in" different fragments (e.g., fluorescence tags, polymers, drugs) into the scaffold arms [1], which can be used to mimic discontinuous protein epitopes [3]. With this in mind, we developed a trifunctional scaffold specifically designed for the combinatorial solid-phase syntheses of protein binders [4]. We utilized solid-phase synthesis, which is a convenient and widely used method in combinatorial chemistry that generates simple mixtures of compounds by splitting and mixing the resin [5] and allows for clean post-reaction work-up. Scaffold 1 (Scheme 1) comprises three propargylamine arms with free, TES-protected, and TIPS-protected arms to facilitate three successive copper(I)-catalyzed azide-alkyne cycloadditions (CuAACs) with different azides; this strategy was first developed by Aucagne's group [6]. Compound $\mathbf{1}$ is assembled on a central rigid core (trimesic acid) that directs the three arms in distinct positions. An additional fluorinated arm enables both the attachment of the scaffold onto the resin and quantitative analyses by ${ }^{19}$ F-NMR [4].
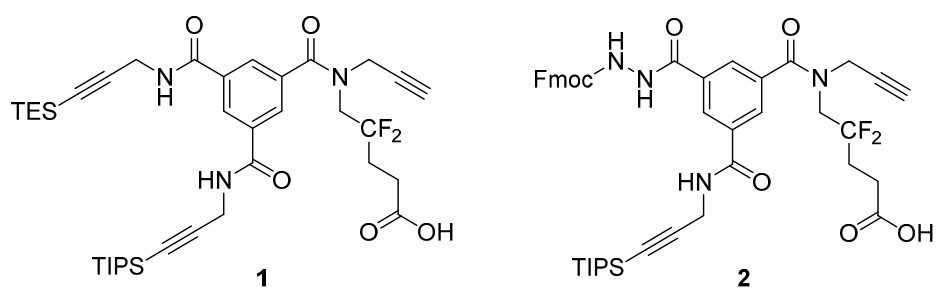

Scheme 1. Previously developed scaffold 1 [4] and scaffold 2 (designed in this study).

Previously, we found that scaffold $\mathbf{1}$ could be improved, specifically in the context of solid-phase combinatorial synthesis. First, only a limited number of azides are commercially available, which limits the size of the libraries and thus the chemical variants. Second, we reported [4] that TES groups are unstable in high copper concentrations, which is problematic when using bulky/hindered azides that often require higher copper loads for complete coupling. Thus, we developed a new scaffold by replacing the TES-protected alkyne arm with a hydrazide moiety (compound 2, Scheme 1). We chose the hydrazide functionality because (i) it can be easily incorporated into the trimesic acid template; (ii) hydrazides can be easily "clicked" onto aldehydes; (iii) a wide variety of aldehydes are commercially available; and (iv) hydrazone and $\mathrm{CuAAC}$ chemistries are, in principle, orthogonal. Here, we report the solution synthesis of scaffold 2 and optimization of its solid-phase derivatization with azides and aldehydes. We also discuss the scope and limitations of this new trifunctional scaffold and, in particular, the compatibility of the $\mathrm{CuAAC}$ and acylhydrazone chemistries in the solid phase.

\section{Results and Discussion}

\subsection{Synthesis in Solution: Preparation of Scaffold $\mathbf{2}$}

We synthesized scaffold $\mathbf{2}$ in solution from diamide $\mathbf{3}$, which was prepared as previously described [4] (Scheme 2). During the amide coupling of diamide 3 and Fmoc-hydrazine [7], using conditions used for the synthesis of scaffold 1 (i.e., PyBrop, DMF, and TEA), we observed the partial deprotection of the 
Fmoc-hydrazide moiety. Consequently, we used ACN to slow the Fmoc deprotection [8] and used the bulkier DIPEA as a base. Heating $\left(60^{\circ} \mathrm{C}, 3 \mathrm{~h}\right)$ was necessary to generate compound 4 , and a TLC analysis of the reaction mixture showed no dibenzofulvene adduct or Fmoc deprotection. After removal of the tert-butyl ester in 50\% TFA/DCM $(v / v)$, we obtained the carboxylic acid 2 in high purity (97\%, HPLC chromatogram in Supplementary Figure S4). Similar to scaffold 1, scaffold 2 presents two alkyne sites in free and TIPS-protected form for CuAAC, an Fmoc-protected hydrazide for the "click-reaction" with aldehydes, and one arm with a carboxylic acid site for attachment to the resin and a $\mathrm{CF}_{2}$ group for ${ }^{19}$ F-NMR quantification.
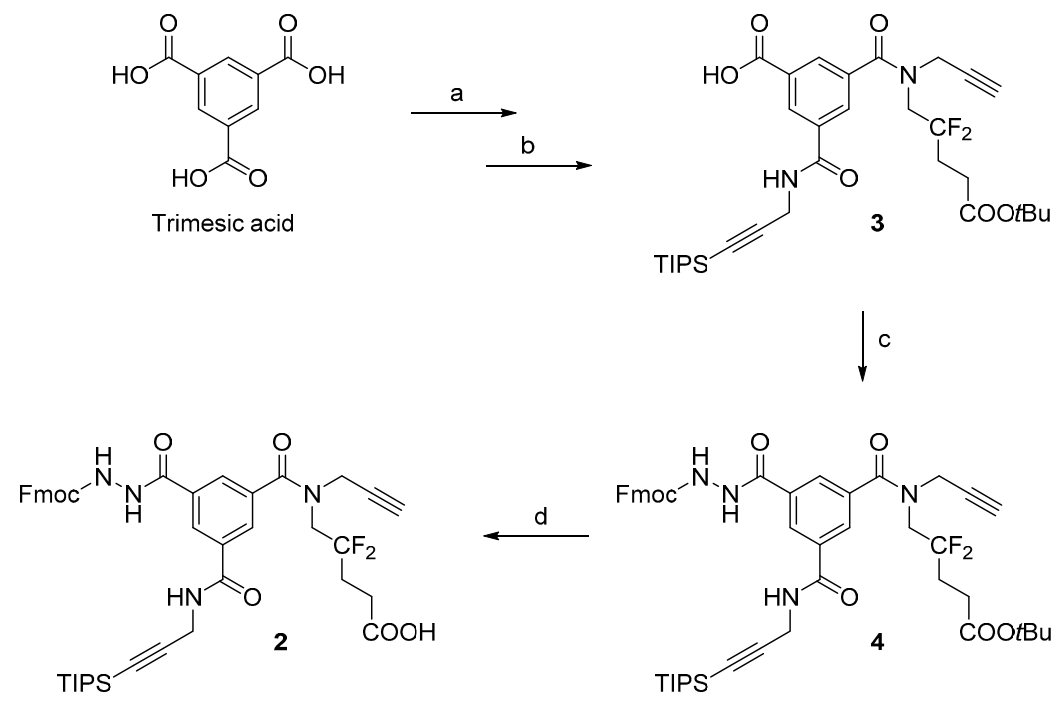

Scheme 2. Synthesis of scaffold 2. Reaction conditions: for the synthesis of $\mathbf{3}$ (steps $(\mathbf{a}, \mathbf{b})$ ), refer to our previous report [4]; (c) Fmoc-hydrazine, DIPEA, PyBroP, ACN, $60{ }^{\circ} \mathrm{C}, 3 \mathrm{~h}$, $57 \%-67 \%$; (d) $50 \%$ TFA/DCM (v/v), r.t., 1 h, 87\%-91\%.

\subsection{Synthesis on Resin: Low Copper Load}

Similar to our previous study [4], we used a PEG-based ChemMatrix resin [9,10] because it swells well in both polar and nonpolar solvents and permits the use of $t \mathrm{BuOH} / \mathrm{H}_{2} \mathrm{O}$ solvent mixtures for CuAAC. We also used the Ramage linker, which can be cleaved in low TFA concentrations (3\%-5\%) to afford terminal amides [11].

To attach scaffold 2 to the resin, for the reasons mentioned above, we again used ACN as the solvent and DIPEA as the base. We estimated the loading of scaffold 2 on the resin by determining the quantity of unreacted scaffold 2 present in the washing solutions (by UV spectrometry, as explained in the Experimental Section). To spare scaffold $\mathbf{2}$ and limit steric hindrance on the resin, we loaded twice as much resin as scaffold 2 , which led to $40 \%-43 \%$ loadings of scaffold 2 in several repeated runs.

As a consequence of this partial loading, free primary amine groups remained on the resin. To inhibit the reaction of these amines with the aldehyde used for acylhydrazone formation, we capped the free amines with Boc-protecting groups (step d, Scheme 3).

The CuAAC on the TIPS-protected alkyne was carried out last because TBAF can cleave Fmoc groups $[8,12]$ and the resulting free hydrazide $\left(\mathrm{R}-\mathrm{CO}-\mathrm{NH}-\mathrm{NH}_{2}\right)$ is unstable (see below). In contrast, the $\mathrm{CuAAC}$ on the free alkyne and the acylhydrazone formation should be interchangeable. However, we 
observed for a model azide that the first CuAAC was significantly faster if performed before the acylhydrazone formation (Scheme S6 and Figure S3).

Following the results from these preliminary experiments, we used the following synthetic order: the first $\mathrm{CuAAC}$ on the free alkyne, acylhydrazone formation, and the second CuAAC on the TIPS-protected alkyne. We then synthesized model compound $\mathbf{8}$ (Scheme 3), and used azides $\mathbf{9}$ and $\mathbf{1 0}$ to determine whether CuAAC with bulky and lipophilic azides reacted on the polar ChemMatrix resin in a $t \mathrm{BuOH} / \mathrm{H}_{2} \mathrm{O}$ solvent mixture.
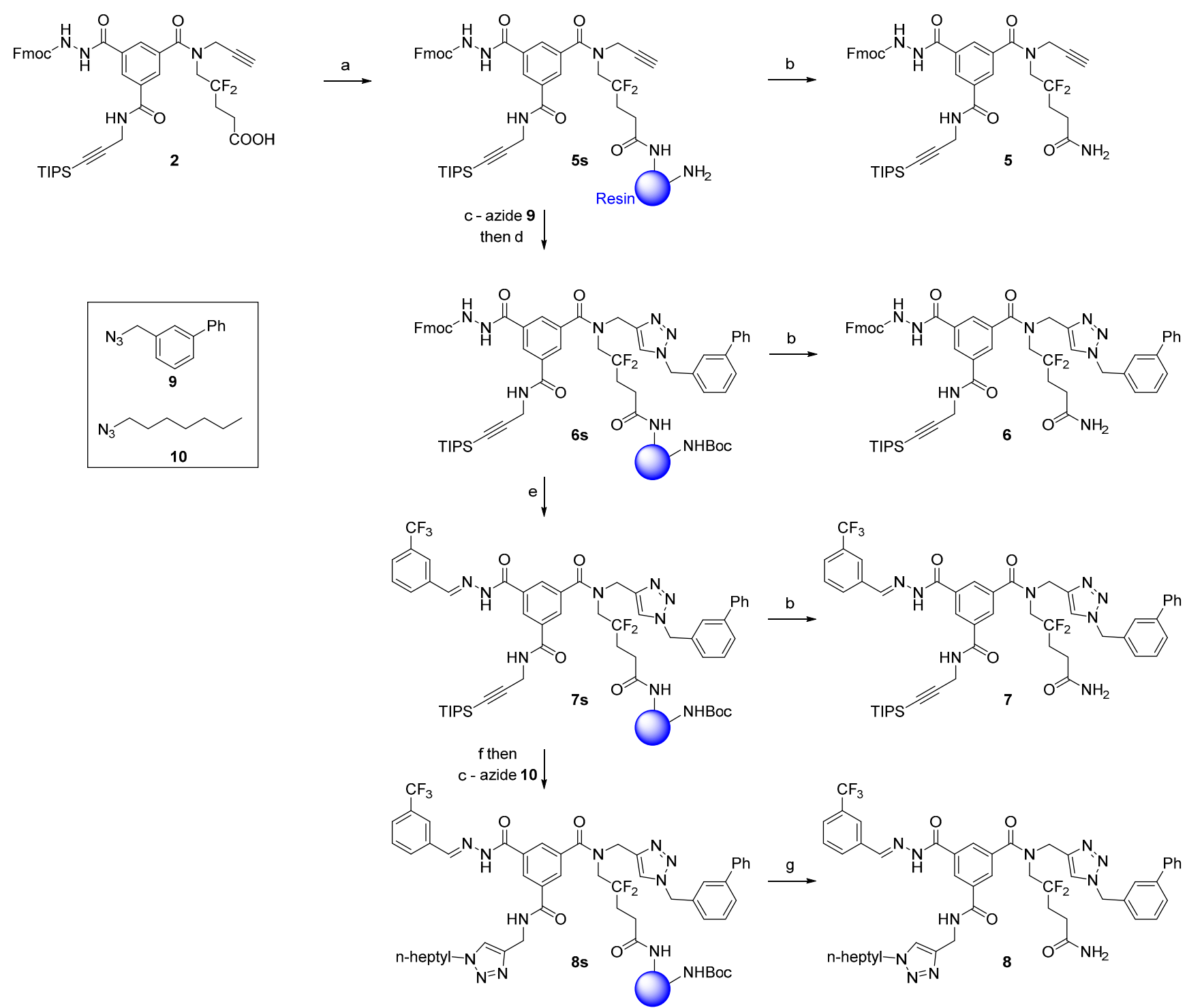

Scheme 3. Solid-phase derivatization of scaffold 2. Reaction conditions: (a) Ramage ChemMatrix (2 eq.), PyBroP (2 eq.), DIPEA (1.5 eq.) in ACN, r.t., 6 h; (b) 5\% TFA/DCM $(v / v)$, r.t., $2 \times 30 \mathrm{~min}$; (c) azide 9 or 10 (5 eq.), sodium ascorbate $\left(0.5\right.$ eq.), $\mathrm{CuSO}_{4} \cdot 5 \mathrm{H}_{2} \mathrm{O}$ (0.1 eq.), tBuOH/water (1:1), r.t., $16 \mathrm{~h}$ for azide 9, $16 \mathrm{~h}+4 \mathrm{~h}$ for azide 10; (d) $\mathrm{Boc}_{2} \mathrm{O}$ (10 eq.), DIPEA (2 eq.), r.t., 3 h; (e) 3-(trifluoromethyl)benzaldehyde (5 eq.), 10\% TEA/ACN (v/v), r.t., 16 h; (f) TBAF (5 eq.), DMF, r.t., $3 \times 1.5$ h; (g) 5\% TFA/DCM (v/v), r.t., $2 \times 30 \mathrm{~min}$, overall crude yield from 2: 24\% OR; gaseous $\mathrm{H}_{2} \mathrm{~S}$ treatment (30 min) followed by $5 \%$ TFA/DCM $(v / v)$, r.t., $2 \times 30 \mathrm{~min}$, overall crude yield from $2: 41 \%$. 
The first low-copper-load CuAAC ( 0.1 equivalent of copper) was completed after $16 \mathrm{~h}$ and produced compound 6 in high purity. Then, we used 20\% piperidine in DMF to remove the Fmoc group from the resin-bound compound $\mathbf{6 s}$. However, cleavage from the resin produced a multi-component mixture, which was unexpected because the Fmoc-deprotection of compound $\mathbf{6}$ in solution rapidly afforded the pure free hydrazide. Performing sequential Fmoc deprotections and acylhydrazone formations from $\mathbf{6 s}$ also afforded multi-component mixtures. We hypothesized that the free hydrazide may have decomposed, and we therefore aimed to trap it with an aldehyde and carry out a one-pot Fmoc-deprotection/acylhydrazone formation. Here, we used triethylamine (TEA) as a base because it deprotects Fmoc groups relatively quickly and does not interfere with the condensation of the free hydrazide with aldehydes. Thus, treating the resin-bound compound $\mathbf{6 s}$ with 3-(trifluoromethyl)benzaldehyde (5 equivalents) in 10\% TEA/ACN $(v / v)$ led to the acylhydrazone 7 in good purity (62\%, HPLC chromatogram in Supplementary Figure S5). This one-step Fmoc-deprotection/acylhydrazone formation has the potential to be a useful tool in "click chemistry", as Fmoc hydrazine can be easily introduced onto carboxylic acids by simple amide coupling and the resulting Fmoc-protected hydrazide can be smoothly converted to acylhydrazone in a single step.

The final product, 8 , was easily obtained by the subsequent removal of the TIPS group with TBAF and a second low-copper-load CuAAC ( 0.1 equivalent of copper). This second $\mathrm{CuAAC}$ was slower than the first and required two treatments ( $16 \mathrm{~h}$ and $4 \mathrm{~h}$ ) to reach completion, possibly due to the slow diffusion of the highly lipophilic and bulky azide $\mathbf{1 0}$ into the polar resin. Crude compound $\mathbf{8}$ had good HPLC purity (71\%, Supplementary Figure S6), and elemental analysis showed an acceptably low amount of copper (100 ppm). ${ }^{19} \mathrm{~F}-\mathrm{NMR}$ analysis [4] was used to determine the quantity of desired compound, and $4.8 \mathrm{mg}$ of crude 8 (theoretically $5.1 \mu \mathrm{mol}$ ) showed $4.8 \mu \mathrm{mol}$ (94\%) of fluorinated compound(s) (Supplementary Figure S11).

In this first experiment, the crude yield of compound 8 was modest (24\%) and lower than expected [4]. However, treatment of the resin with hydrogen sulfide prior to the final cleavage significantly improved the yields (see below), and we obtained $39 \mathrm{mg}$ of crude compound 8 (41 $\mu \mathrm{mol}, 41 \%$, if pure) by starting from $82 \mathrm{mg}$ of scaffold $2(100 \mu \mathrm{mol})$.

\subsection{Synthesis on Resin: High Copper Load}

Large/bulky azido ligands often require high copper concentrations to react efficiently with alkynes [13]. However, we previously [4] observed that high copper loads led to the deprotection of TES groups, which could be problematic when using scaffold 1 (Scheme 1) in CuAAC on the first unprotected alkyne. In these cases, catalytic amounts of copper could lead to incomplete coupling, whereas higher copper loads could potentially generate double cycloaddition products at the free and TES-protected alkyne arms.

In order to verify whether our new scaffold tolerated high copper loads during both CuAACs, we re-synthesized compound $\mathbf{8}$ (Scheme 3 ) using one equivalent of copper sulfate in each CuAAC. However, after the final cleavage from the resin, we obtained a mixture containing compound $\mathbf{8}$ and carboxylic acid 12 (structure in Scheme 4A) in a 1:1 ratio (Figure S7A).

Because the only difference between the two syntheses of 8 was the high copper load, we suspected that residual copper ions retained in the PEG-resin participated in the degradation of 8 . Previously, we reported that treatment of the final compounds with gaseous hydrogen sulfide led to the formation of insoluble copper salts (presumably $\mathrm{CuS}$ ) that could be easily filtered out [4]. To investigate whether 
treating the resin-bound compound directly with hydrogen sulfide could prevent copper ions from interacting with the target compounds and to understand when the acylhydrazone hydrolysis takes place, we repeated the synthesis of compound 8 using low ( 0.1 equivalent) and high ( 1 equivalent) loads of copper sulfate in each $\mathrm{CuAAC}$. We then treated or did not treat the resin with hydrogen sulfide after each $\mathrm{CuAAC}$ and analyzed the intermediate compounds 6 and 7 by HPLC. While high copper concentrations were problematic, we were unable to distinguish whether the copper ions participated only in acylhydrazone degradation during cleavage from the resin or if they also interfered with acylhydrazone formation. However, we observed that the presence of copper (II) ions in solution has a negligible impact on the condensation reaction between benzhydrazide and 3-(trifluoromethyl)benzaldehyde (Supplementary Materials, Figure S2 and Table S1). In either case, hydrogen sulfide efficiently "inactivated" the copper ions present in the resin. Thus, synthesis using one equivalent of copper and treatment of the resin with hydrogen sulfide after each $\mathrm{CuAAC}$ generated compound $\mathbf{8}$ in purity comparable to synthesis with a low copper load (64\% purity, Supplementary Figure S7B). Furthermore, compound 8 was obtained in a higher crude yield (47\%) and elemental analysis verified that the content of copper in the final crude compound $\mathbf{8}$ was low (15 ppm) despite the use of a higher copper load.

\subsection{Synthesis on Resin: Scope and Limitations}

The scope of the CuAAC [14,15], the condensation between hydrazines and aldehydes, the Boc-protection of amines, and the deprotection of TIPS group by TBAF are well studied and compatible with a wide range of functional groups. The main limitations of our chemistry stem from acidic cleavage (5\% TFA/DCM) that precludes the presence of acid-labile groups (e.g., acetal) and the use of hydrogen sulfide gas. Hydrogen sulfide in solution is a reducing agent that reduces azide functions in water [16], and is also a nucleophilic species that reacts with disulfides [17].

Additionally, we studied whether different aldehydes are compatible with the presented chemistry. We chose 1-butyraldehyde and 4-methoxybenzaldehyde as models of aliphatic and electron-rich aldehydes, respectively (Scheme 4).

\subsubsection{Aliphatic Aldehydes}

First, we observed that aliphatic acylhydrazone was unstable in the acidic cleavage mixture (5\% TFA/DCM) and quickly hydrolyzed. We attempted to solve this problem by reducing the acylhydrazone group to the hydrazide, synthesizing the substituted hydrazide 11s from the resin-bound scaffold $\mathbf{5 s}$ (Scheme 4A). The Fmoc-protected hydrazide 5s was reacted with 1-butyraldehyde (low copper load), and the resulting acylhydrazone was reduced to the substituted hydrazide 11s directly on the resin with sodium borohydride because milder reducing agents (i.e., sodium triacetoxyborohydride and sodium cyanoborohydride) were inefficient. Upon cleavage from the resin, we obtained the substituted hydrazide 11 in good purity (70\% by HPLC, Supplementary Figure S8). It was important that the acylhydrazide formation was performed before the first $\mathrm{CuAAC}$, as the presence of residual copper in the resin results in a messy reduction (appearance of a black colloid when sodium borohydride is added) [18].

The synthesis was completed following the protocol described for compound $\mathbf{8}$ under low copper loads. However, after final cleavage from the resin, the substituted hydrazide moiety was fully hydrolyzed and we obtained only the benzoic acid derivative 12 (Supplementary Figure S9). Because compound 11 was 
stable in acidic conditions, it appears that copper ions participate in the hydrolysis, supporting previous reports that $\mathrm{Cu}$ (II) ions catalyze the apparent hydrolysis of poly-substituted aliphatic hydrazides [19-21] by initiating aerobic oxidation of the hydrazide moiety [21]. Hence, we verified that $\mathrm{Cu}(\mathrm{II})$ ions indeed promote the hydrolysis of $N^{\prime}$-butylbenzohydrazide in aqueous solution (Supporting Information, Figure S1). Treating the resin with gaseous hydrogen sulfide after each CuAAC did not lessen this effect, and the substituted aliphatic acylhydrazide was still hydrolyzed.
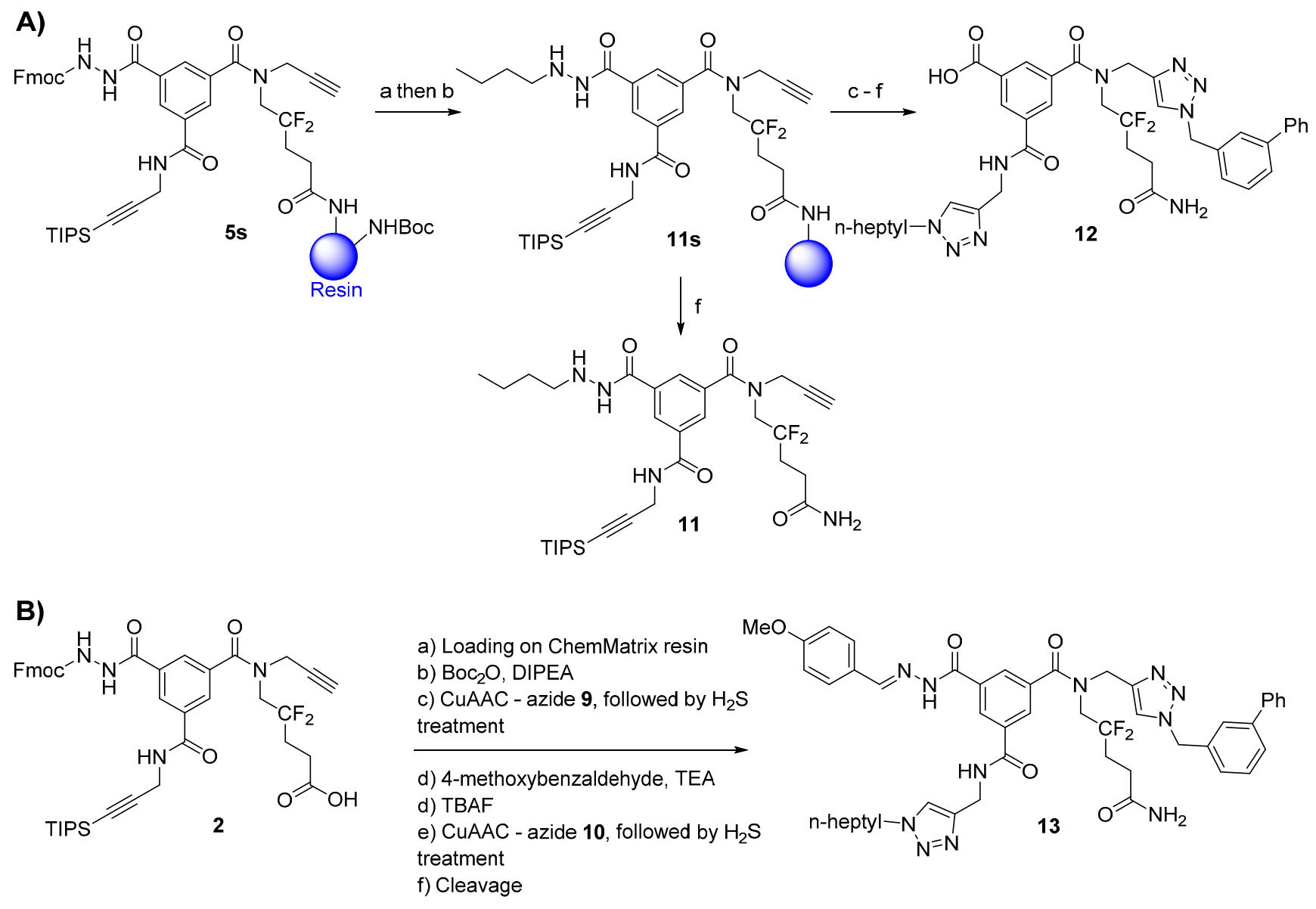

Scheme 4. Study of the scope of the reaction. (A) Reactions with the aliphatic aldehyde. Reaction conditions: (a) 1-butyraldehyde (5 eq.), 10\% TEA/ACN (v/v), r.t., 16 h; (b) $\mathrm{NaBH}_{4}$ (10 eq.), dry EtOH, r.t., 3 h; (c) azide 9 ( 5 eq.), sodium ascorbate (0.5 eq.), $\mathrm{CuSO}_{4} \cdot 5 \mathrm{H}_{2} \mathrm{O}$ (0.1 eq.), $t \mathrm{BuOH} /$ water (1:1), r.t., 16 h; (d) TBAF (5 equiv.), DMF, r.t., $3 \times 1.5$ h; (e) azide 10 (5 eq.), sodium ascorbate (0.5 eq.), $\mathrm{CuSO}_{4} \cdot 5 \mathrm{H}_{2} \mathrm{O}$ (0.1 eq.), $t \mathrm{BuOH} /$ water (1:1), r.t., $16 \mathrm{~h}+4 \mathrm{~h}$; (f) $5 \% \mathrm{TFA} / \mathrm{DCM}(v / v)$, r.t., $2 \times 30 \mathrm{~min}$; (B) Reactions with the aromatic aldehyde. The reaction conditions are identical to those in Scheme 3 (steps a, c-f), apart from the final cleavage from the resin: $5 \% \mathrm{TFA} / \mathrm{DCM}(v / v), 2 \% \mathrm{TIS}(v / v)$, r.t., $2 \times 30 \mathrm{~min}$, overall crude yield from 2: $43 \%$.

\subsubsection{Electron-Rich Aromatic Aldehydes}

Next, we investigated the synthetic compatibility of the electron-rich 4-methoxybenzaldehyde. Electron-rich aromatic aldehydes are less electrophilic than aliphatic or electron-poor aromatic aldehydes and react more slowly with nucleophiles. Compared to the low copper load protocol shown in Scheme 3, 
we added triisopropylsilane (TIS) to scavenge the carbocations formed in the acidic medium, as these carbocations may add to the electron-rich anisole ring (Scheme 4B). After each CuAAC, the resin was treated with gaseous $\mathrm{H}_{2} \mathrm{~S}$, and compound 13 was obtained as the major product but in lower purity levels than compound 8 (HPLC chromatogram in Supplementary Figure S10). The lower purity of crude 13 prompted us to investigate its stability in an acidic non-aqueous solvent (5\% TFA/DCM, i.e., cleavage mixture), an acidic aqueous solvent (50\% ACN/water + 0.1\% TFA, i.e., HPLC solvent), and the HPLC solvent with $\mathrm{Cu}$ (II) ions (one equivalent of copper sulfate pentahydrate). These experiments revealed that $1 \mathrm{~h}$ treatment in 5\% TFA/DCM did not affect compound 13, whereas the two aqueous conditions decomposed $\mathbf{1 3}$ to generate different hydrolysis products (see discussion in Supporting Materials, Table S2 and Scheme S5).

\subsection{Final Remarks}

We report here observations that could be useful to chemists combining the CuAAC and acylhydrazone chemistry or solid-phase CuAAC.

First, the aliphatic acylhydrazide $\mathbf{1 1}$ was hydrolyzed to the carboxylic acid derivative during the synthesis. Using a model aliphatic acylhydrazide (Supporting Materials, Figure S1), we observed that such hydrolysis occurs rapidly in aqueous solution with $\mathrm{Cu}(\mathrm{II})$ ions $\left(\mathrm{CuSO}_{4} \cdot 5 \mathrm{H}_{2} \mathrm{O}\right)$ and more slowly in the $\mathrm{CuAAC}$ conditions used here $\left(\mathrm{CuSO}_{4} \cdot 5 \mathrm{H}_{2} \mathrm{O}\right.$ and sodium ascorbate). Thus, $\mathrm{CuAAC}$ in aqueous medium should not be performed in the presence of an aliphatic acylhydrazide moiety as the acylhydrazide could be hydrolyzed.

Second, the presence of copper ions (presumably trapped in the PEG-resin) affected hydrazone reduction and promoted the hydrolysis of aromatic acylhydrazone during the cleavage from the resin, hindering solid-phase synthesis. Here, we demonstrated that treatment of the resin with gaseous hydrogen sulfide during the synthesis efficiently inactivated the copper ions, which could potentially inactivate other interfering metal ions [22].

\section{Experimental Section}

\subsection{General Information}

Unless otherwise stated, reagents and solvents used in this study were obtained from commercial suppliers and used without purification. EtOH was dried with 3- $\AA$ molecular sieves, as described in the literature [23]. The solvents were evaporated at $55{ }^{\circ} \mathrm{C}$ and $2 \mathrm{kPa}$, and the products were dried over phosphorus pentoxide at r. t. and $13 \mathrm{~Pa}$. TLC were run on aluminum plates coated with silica gel $\left(60 \mathrm{~F}_{254}\right.$, Merck, Darmstadt, Germany). The compounds were visualized by exposure to UV light at $254 \mathrm{~nm}$. Flash chromatography purifications were carried out on silica gel (40-63 $\mu \mathrm{m}$, Fluka, Darmstadt, Germany). Analytical RP-HPLC chromatography of the compounds were carried out on a Waters HPLC system (Waters 1525 Binary HPLC Pump and Waters 2787 Dual $\lambda$ Absorbance Detector, Milford, MA, USA) using Nucleosil 120-5 C8 column $(250 \times 4.6 \mathrm{~mm}, 5 \mu \mathrm{m}$, Watrex, Praha, Czech Republic) at a flow rate of $1 \mathrm{~mL} / \mathrm{min}$. Solvent A: $0.1 \%$ TFA $(v / v)$ in $\mathrm{H}_{2} \mathrm{O}$. Solvent B: $0.1 \%$ TFA $(v / v)$ in $\mathrm{CH}_{3} \mathrm{CN}$. The following gradients were used: $\mathrm{t}=0 \mathrm{~min} / 50 \% \mathrm{~B}, \mathrm{t}=30 \mathrm{~min} / 100 \% \mathrm{~B}, \mathrm{t}=31 \mathrm{~min} / 50 \% \mathrm{~B}$ (Method $\mathrm{A}$ ), or $\mathrm{t}=0 \mathrm{~min} / 20 \% \mathrm{~B}, \mathrm{t}=30 \mathrm{~min} / 100 \% \mathrm{~B}, \mathrm{t}=31 \mathrm{~min} / 20 \% \mathrm{~B}($ Method $\mathrm{B})$. The compounds were detected at 
218 and $254 \mathrm{~nm}$. The purity of a given compound was determined by integration of the whole spectra at $218 \mathrm{~nm}$. Preparative RP-HPLC chromatography of compounds was carried on the same Waters HPLC system but using a Vydac 214TP101522 C4 column $(250 \times 22 \mathrm{~mm}, 10-15 \mu \mathrm{m}$, Columbia, MD, USA) at a flow rate of $9 \mathrm{~mL} / \mathrm{min}$. The gradients and the detection were the same as used in analytical HPLC. HRMS spectra were obtained on an LTQ-orbitrap XL FTMS mass spectrometer (Thermo Fisher, Bremen, Germany) in electrospray ionization mode or in case HRMS (EI) on GCT Premier (Waters). ${ }^{1} \mathrm{H}-$ and ${ }^{13} \mathrm{C}-\mathrm{NMR}$ spectra were measured on a Bruker AVANCE-600 instrument $\left({ }^{1} \mathrm{H}\right.$ at $600 \mathrm{MHz}$ and ${ }^{13} \mathrm{C}$ at 150.9 MHz frequency, Rheinstatten, Germany) or AVANCE-500 instrument $\left({ }^{1} \mathrm{H}-\right.$ at $500 \mathrm{MHz}$ and ${ }^{13} \mathrm{C}$ at $125.8 \mathrm{MHz}$ frequency, Rheinstatten, Germany) in DMSO- $d_{6}$. The 2D-H,H-COSY, 2D-H,C-HSQC and 2D-H,C-HMBC spectra were recorded and used for the structural assignment of proton and carbon signals. The presence of amide bonds and bulky arms leads to a slow rotation of the trimesic acid substituents and results in line broadening of many signals. The increasing temperature leads to the narrowing of signals or to the coalescence of doubled peaks but some signals could not be detected even at $100{ }^{\circ} \mathrm{C}$ (in particular signals from the tertiary amide arm). The ${ }^{19} \mathrm{~F}-\mathrm{NMR}$ spectrum was measured on a Bruker AVANCE-500 instrument (Rheinstatten, Germany) at 470.4 MHz. For the quantitative estimation of the fluorine containing compounds the ${ }^{19} \mathrm{~F}$ proton-decoupled spectra with suppression of heteronuclear NOE during relaxation delay ( $3 \mathrm{~s}$ ) were measured. A known amount of $\mathrm{BrCF}_{2} \mathrm{COOEt}$ was added to the measured solution and used as a standard for quantitative analysis. IR spectra were recorded on Bruker IFS 55 Equinox apparatus (Rheinstatten, Germany). UV-Vis spectrometry was carried out on a Perkin Elmer Lambda 25 system (Norwalk, CT, USA).

\subsection{Syntheses}

\subsubsection{Compound 4}

A suspension of compound 3 [4] (5.6 g, $8.8 \mathrm{mmol})$, PyBrop (6.2 g, $13.3 \mathrm{mmol}$, 1.5 eq.), Fmoc-hydrazine [7] (6.7 g, $26.3 \mathrm{mmol}, 3.0$ eq.) and DIPEA (3.08 mL, $17.7 \mathrm{mmol}, 2.0$ eq.) in ACN (150 mL) was stirred at $60{ }^{\circ} \mathrm{C}$. After $3 \mathrm{~h}$, the reaction was cooled to r.t. and then filtered to remove excess Fmoc-hydrazine. The filtrate was evaporated, suspended in EtOAc $(60 \mathrm{~mL})$, and filtered again to remove the remaining Fmoc-hydrazine. The EtOAc filtrate was poured into a separatory funnel, washed a saturated $\mathrm{NH}_{4} \mathrm{Cl}$ solution $(2 \times 30 \mathrm{~mL})$ and brine $(50 \mathrm{~mL})$, dried over anhydrous sodium sulfate, filtered, and evaporated to give $8.5 \mathrm{~g}$ of brown oil. The crude product was purified by flash chromatography on silica (200 g of silica, elution with a linear gradient-5\% to $65 \%$ - of EtOAc in toluene), resulting in compound 4 as a pale yellow solid ( $4.5 \mathrm{~g}, 58 \%, 57 \%-67 \%$ in repeated runs; $\mathrm{R}_{f}=0.3$ in $7: 3$ toluene/EtOAc).

Analytical HPLC: $\left(\right.$ Method A) $t_{\mathrm{R}}=24.5 \mathrm{~min} .{ }^{1} \mathrm{H}-\mathrm{NMR}\left(500 \mathrm{MHz}\right.$; DMSO- $\left.d_{6} ; \mathrm{T}=100{ }^{\circ} \mathrm{C}\right): \delta_{\mathrm{H}} 1.06(21 \mathrm{H}$, m, $\left.\mathrm{Si}\left(\mathrm{CH}\left(\mathrm{CH}_{3}\right)_{2}\right)_{3}\right), 1.41\left(9 \mathrm{H}, \mathrm{s}, \mathrm{C}\left(\mathrm{CH}_{3}\right)_{3}\right), 2.22\left(2 \mathrm{H}, \mathrm{m},-\mathrm{CH}_{2}-\right), 2.38\left(2 \mathrm{H}, \mathrm{t},{ }^{3} \mathrm{~J}_{\mathrm{HH}}=7.5 \mathrm{~Hz},-\mathrm{CH}_{2}-\mathrm{CO}\right)$, $3.12\left(1 \mathrm{H}, \mathrm{t},{ }^{4} J_{\mathrm{HH}}=2.4 \mathrm{~Hz},-\mathrm{C} \equiv \mathrm{CH}\right), 4.03\left(2 \mathrm{H}, \mathrm{t},{ }^{3} J_{\mathrm{HF}}=14.2 \mathrm{~Hz},-\mathrm{CH}_{2}-\mathrm{N}\right), 4.18\left(2 \mathrm{H}, \mathrm{d},{ }^{3} J_{\mathrm{HH}}=5.6 \mathrm{~Hz}\right.$, $\left.-\mathrm{CH}_{2}-\mathrm{N}\right), 4.25\left(2 \mathrm{H}, \mathrm{d},{ }^{4} \mathrm{~J}_{\mathrm{HH}}=2.4 \mathrm{~Hz},-\mathrm{CH}_{2}-\mathrm{N}\right), 4.27\left(1 \mathrm{H}, \mathrm{t},{ }^{3} \mathrm{~J}_{\mathrm{HH}}=7.0 \mathrm{~Hz},>\mathrm{CH}-\mathrm{Fmoc}\right), 4.40(2 \mathrm{H}, \mathrm{d}$, ${ }^{3} J_{\mathrm{HH}}=7.0 \mathrm{~Hz},-\mathrm{CH}_{2}-\mathrm{O}$ Fmoc $), 7.30\left(2 \mathrm{H}, \mathrm{t},{ }^{3} J_{\mathrm{HH}}=7.5 \mathrm{~Hz}, 2 \times \mathrm{CH}_{\text {arom }}\right), 7.40\left(2 \mathrm{H}, \mathrm{t},{ }^{3} J_{\mathrm{HH}}=7.5 \mathrm{~Hz}\right.$, $\left.2 \times \mathrm{CH}_{\text {arom }}\right), 7.70\left(2 \mathrm{H}, \mathrm{d},{ }^{3} \mathrm{~J}_{\mathrm{HH}}=7.5 \mathrm{~Hz}, 2 \times \mathrm{CH}_{\text {arom }}\right), 7.84\left(2 \mathrm{H}, \mathrm{d},{ }^{3} \mathrm{~J}_{\mathrm{HH}}=7.5 \mathrm{~Hz}, 2 \times \mathrm{CH}_{\text {arom }}\right), 8.04(1 \mathrm{H}$, $\left.\mathrm{t},{ }^{3} J_{\mathrm{HH}}=1.5 \mathrm{~Hz}, \mathrm{CH}_{\text {arom }}\right), 8.05\left(1 \mathrm{H}, \mathrm{t},{ }^{3} \mathrm{JHH}_{\mathrm{HH}}=1.5 \mathrm{~Hz}, \mathrm{CH}_{\text {arom }}\right), 8.45\left(1 \mathrm{H}, \mathrm{t},{ }^{3} J_{\mathrm{HH}}=1.5 \mathrm{~Hz}, \mathrm{CH}_{\text {arom }}\right), 8.87(1 \mathrm{H}$,

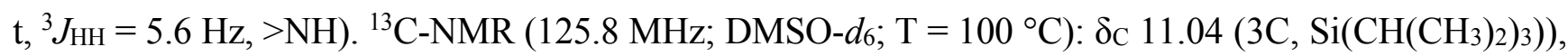


$18.56\left(6 \mathrm{C}, \mathrm{Si}\left(\mathrm{CH}\left(\mathrm{CH}_{3}\right)_{2}\right)_{3}\right), 27.99\left(3 \mathrm{C},-\mathrm{C}\left(\mathrm{CH}_{3}\right)_{3}\right), 28.22\left(1 \mathrm{C},-\mathrm{CH}_{2}-\right), 29.94\left(1 \mathrm{C},-\mathrm{CH}_{2}-\mathrm{N}\right), 30.26(1 \mathrm{C}$, $\left.\mathrm{t},{ }^{2} J_{\mathrm{CF}}=24.4 \mathrm{~Hz},-\mathrm{CH}_{2}-\right), 39.40\left(1 \mathrm{C},-\mathrm{CH}_{2}-\mathrm{N}\right), 47.05(1 \mathrm{C},>\mathrm{CH}-\mathrm{Fmoc}), 49.45\left(1 \mathrm{C},-\mathrm{CH}_{2}-\mathrm{N}\right), 66.75$ $\left(1 \mathrm{C},-\mathrm{CH}_{2}-\mathrm{O}\right.$ Fmoc $), 75.72(1 \mathrm{C}, \equiv \mathrm{C}-\mathrm{H}), 78.60(1 \mathrm{C}, \equiv \mathrm{C}-), 80.40\left(1 \mathrm{C}, \mathrm{C}\left(\mathrm{CH}_{3}\right)_{3}\right), 82.36(1 \mathrm{C}, \equiv \mathrm{C}-), 105.64$ $(1 \mathrm{C}, \equiv \mathrm{C}-), 120.13\left(2 \mathrm{C}, 2 \times \mathrm{CH}_{\text {arom }}\right), 123.47\left(1 \mathrm{C}, \mathrm{t},{ }^{1} J_{\mathrm{CF}}=244.5 \mathrm{~Hz},>\mathrm{CF}_{2}\right), 125.34\left(2 \mathrm{C}, 2 \times \mathrm{CH}_{\text {arom }}\right), 127.20$ (2C, $\left.2 \times \mathrm{CH}_{\text {arom }}\right), 127.79$ (2C, $\left.2 \times \mathrm{CH}_{\text {arom}}\right), 128.19$ (1C, $\left.\mathrm{CH}_{\text {arom }}\right), 128.21$ (1C, $\left.\mathrm{CH}_{\text {arom }}\right), 128.56$ (1C, $\left.\mathrm{CH}_{\text {arom}}\right), 133.68$ (1C, $\left.\mathrm{C}_{\text {arom}}\right), 135.38$ (1C, $\left.\mathrm{C}_{\text {arom }}\right), 135.88$ (1C, $\left.\mathrm{C}_{\text {arom}}\right), 141.02$ (2C, $\left.\mathrm{C}_{\text {arom }}\right), 143.94$ (2C, Carom), 156.32 (1C, -CO-NH), 164.96 (1C, -CO-NH), 170.39 (1C, -CO-NH), 170.74 (1C, -CO-O) $\left(-\mathrm{CO}-\mathrm{N}<\right.$ missing). IR (KBr) $v_{\max }=3420(\mathrm{~m}, \mathrm{vbr}, \mathrm{NH}), 3306(\mathrm{~m}, \mathrm{C} \equiv \mathrm{C}-\mathrm{H}), 2944$ (s, CH3 TIPS), 2177 (w, $\mathrm{C} \equiv \mathrm{C}), 1731,1657,1526(\mathrm{~s}, \mathrm{br}, \mathrm{C}=\mathrm{O})$. HRMS $(\mathrm{m} / \mathrm{z})$ : calcd for $\mathrm{C}_{48} \mathrm{H}_{58} \mathrm{~F}_{2} \mathrm{~N}_{4} \mathrm{NaO}_{7} \mathrm{Si}^{+}[\mathrm{M}+\mathrm{Na}]^{+}$ 891.39350 , found 891.39383 .

\subsubsection{Scaffold 2}

TFA $(10 \mathrm{~mL})$ was added to a solution of $4(4.4 \mathrm{~g}, 5.1 \mathrm{mmol})$ in DCM $(10 \mathrm{~mL})$ at $0{ }^{\circ} \mathrm{C}$. The reaction mixture was allowed to reach r.t. and was stirred for $1 \mathrm{~h}$. The reaction mixture was co-evaporated with toluene to afford $5.6 \mathrm{~g}$ of brownish foam. The crude product was purified by flash chromatography on silica (200 g of silica, elution with a linear gradient $-0 \%$ to $60 \%$ over $40 \mathrm{~min}$ - of $1 \% \mathrm{AcOH} / \mathrm{EtOAc}$ $(v / v)$ in toluene), resulting in compound 2 as a beige solid (3.6 g, 87\%, 87\%-91\% in repeated runs; $\mathrm{R}_{f}=0.3$ in $1 \% \mathrm{AcOH} / \mathrm{EtOAc}(v / v)$ in toluene $1: 1)$.

Analytical HPLC: (Method A) $t_{\mathrm{R}}=17.9 \mathrm{~min} .{ }^{1} \mathrm{H}-\mathrm{NMR}\left(500 \mathrm{MHz}\right.$; DMSO- $\left.d_{6} ; \mathrm{T}=100{ }^{\circ} \mathrm{C}\right): \delta_{\mathrm{H}} 1.06$ $\left(21 \mathrm{H}, \mathrm{m}, \mathrm{Si}\left(\mathrm{CH}\left(\mathrm{CH}_{3}\right)_{2}\right)_{3}\right), 2.24\left(2 \mathrm{H}, \mathrm{m},-\mathrm{CH}_{2}-\right), 2.42\left(2 \mathrm{H}, \mathrm{t},{ }^{3} \mathrm{~J}_{\mathrm{HH}}=7.5 \mathrm{~Hz},-\mathrm{CH}_{2}-\mathrm{CO}\right), 3.12(1 \mathrm{H}, \mathrm{t}$, $\left.{ }^{4} J_{\mathrm{HH}}=2.4 \mathrm{~Hz},-\mathrm{C} \equiv \mathrm{CH}\right), 4.04\left(2 \mathrm{H}, \mathrm{t},{ }^{3} J_{\mathrm{HF}}=14.5 \mathrm{~Hz},-\mathrm{CH}_{2}-\mathrm{N}\right), 4.18\left(2 \mathrm{H}, \mathrm{d},{ }^{3} J_{\mathrm{HH}}=5.6 \mathrm{~Hz},-\mathrm{CH}_{2}-\mathrm{N}\right)$, $4.25\left(2 \mathrm{H}, \mathrm{d},{ }^{4} \mathrm{~J}_{\mathrm{HH}}=2.4 \mathrm{~Hz},-\mathrm{CH}_{2}-\mathrm{N}\right), 4.27\left(1 \mathrm{H}, \mathrm{t},{ }^{3} \mathrm{~J}_{\mathrm{HH}}=7.0 \mathrm{~Hz},>\mathrm{CH}-\mathrm{Fmoc}\right), 4.40\left(2 \mathrm{H}, \mathrm{d},{ }^{3} \mathrm{~J}_{\mathrm{HH}}=7.0 \mathrm{~Hz}\right.$, $-\mathrm{CH}_{2}-\mathrm{O}$ Fmoc), $7.30\left(2 \mathrm{H}, \mathrm{t},{ }^{3} J_{\mathrm{HH}}=7.5 \mathrm{~Hz}, 2 \times \mathrm{CH}_{\text {arom }}\right), 7.40\left(2 \mathrm{H}, \mathrm{t},{ }^{3} J_{\mathrm{HH}}=7.5 \mathrm{~Hz}, 2 \times \mathrm{CH}_{\text {arom }}\right), 7.71$ $\left(2 \mathrm{H}, \mathrm{d},{ }^{3} \mathrm{~J}_{\mathrm{HH}}=7.5 \mathrm{~Hz}, 2 \times \mathrm{CH}_{\text {arom }}\right), 7.84\left(2 \mathrm{H}, \mathrm{d},{ }^{3} \mathrm{~J}_{\mathrm{HH}}=7.5 \mathrm{~Hz}, 2 \times \mathrm{CH}_{\text {arom }}\right), 8.04\left(1 \mathrm{H}, \mathrm{t},{ }^{3} J_{\mathrm{HH}}=1.5 \mathrm{~Hz}\right.$, $\left.\mathrm{CH}_{\text {arom }}\right), 8.06\left(1 \mathrm{H}, \mathrm{t},{ }^{3} \mathrm{~J}_{\mathrm{HH}}=1.5 \mathrm{~Hz}, \mathrm{CH}_{\text {arom }}\right), 8.44\left(1 \mathrm{H}, \mathrm{t},{ }^{3} \mathrm{~J}_{\mathrm{HH}}=1.5 \mathrm{~Hz}, \mathrm{CH}_{\text {arom }}\right), 8.88\left(1 \mathrm{H}, \mathrm{t},{ }^{3} J_{\mathrm{HH}}=5.6 \mathrm{~Hz}\right.$,

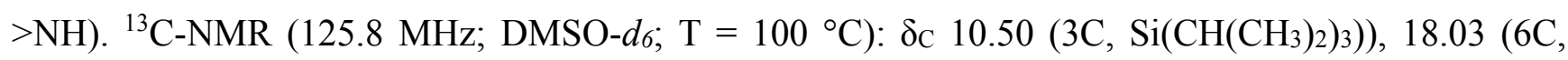
$\left.\mathrm{Si}\left(\mathrm{CH}\left(\mathrm{CH}_{3}\right)_{2}\right)_{3}\right), 26.21\left(1 \mathrm{C}, \mathrm{t},{ }^{3} J_{\mathrm{CF}}=5.2 \mathrm{~Hz},-\mathrm{CH}_{2}-\right), 29.40\left(1 \mathrm{C},-\mathrm{CH}_{2}-\mathrm{N}\right), 29.74\left(1 \mathrm{C}, \mathrm{t},{ }^{2} J_{\mathrm{CF}}=24.2 \mathrm{~Hz}\right.$, $\left.-\mathrm{CH}_{2}-\right), 38.81\left(1 \mathrm{C},-\mathrm{CH}_{2}-\mathrm{N}\right), 46.52(1 \mathrm{C},>\mathrm{CH}-\mathrm{Fmoc}), 66.21\left(1 \mathrm{C},-\mathrm{CH}_{2}-\mathrm{O}\right.$ Fmoc $), 75.21(1 \mathrm{C}, \equiv \mathrm{C}-\mathrm{H})$, $78.10(1 \mathrm{C}, \equiv \mathrm{C}-), 81.83(1 \mathrm{C}, \equiv \mathrm{C}-), 105.12(1 \mathrm{C}, \equiv \mathrm{C}-), 119.60\left(2 \mathrm{C}, 2 \times \mathrm{CH}_{\text {arom }}\right), 124.00(1 \mathrm{C}, \mathrm{t}$, $\left.{ }^{1} J_{\mathrm{CF}}=244.5 \mathrm{~Hz},>\mathrm{CF}_{2}\right), 124.81\left(2 \mathrm{C}, 2 \times \mathrm{CH}_{\text {arom }}\right), 126.66\left(2 \mathrm{C}, 2 \times \mathrm{CH}_{\text {arom }}\right), 127.25\left(2 \mathrm{C}, 2 \mathrm{x} \mathrm{CH}_{\text {arom }}\right)$, 127.64 (1C, $\left.\mathrm{CH}_{\text {arom }}\right), 127.68$ (1C, $\left.\mathrm{CH}_{\text {arom }}\right), 128.02$ (1C, $\left.\mathrm{CH}_{\text {arom }}\right), 133.14$ (1C, Carom), 134.84 (1C, Carom), 135.35 (1C, $\left.\mathrm{C}_{\text {arom }}\right), 140.49$ (2C, $\left.2 \times \mathrm{C}_{\text {arom }}\right), 143.41\left(2 \mathrm{C}, 2 \times \mathrm{C}_{\text {arom }}\right), 155.79$ (1C, $\left.-\mathrm{CO}-\mathrm{NH}\right), 164.43$ (1C, $-\mathrm{CO}-\mathrm{NH}), 169.86(1 \mathrm{C},-\mathrm{CO}-\mathrm{NH}), 172.19(1 \mathrm{C},-\mathrm{COOH})\left(-\mathrm{CO}-\mathrm{N}<\right.$ and one $-\mathrm{CH}_{2}-\mathrm{N}$ missing $) . \mathrm{IR}(\mathrm{KBr})$ $\nu_{\max }=3294$ (m, br, C $\equiv \mathrm{C}-\mathrm{H}$ and NH), 2944 (s, CH3 TIPS), 2178 (w, C $\left.\equiv \mathrm{C}\right), 1723,1657,1528$ (s, br, C=O). HRMS $(m / z)$ : calcd for $\mathrm{C}_{44} \mathrm{H}_{49} \mathrm{~F}_{2} \mathrm{~N}_{4} \mathrm{O}_{7} \mathrm{Si}^{-}[\mathrm{M}-\mathrm{H}]^{-}$811.33441, found 811.33420.

\subsubsection{Compound 5}

Ramage ChemMatrix ${ }^{\circledR}$ resin $(667 \mathrm{mg}, 200 \mu \mathrm{mol}, 0.3 \mathrm{mmol} / \mathrm{g}$, Aldrich 727792) with free amino groups was swelled in $\mathrm{MeOH}, \mathrm{DCM}$, and ACN (10 minutes each, using $10 \mathrm{~mL}$ of solvent/g of resin) in a fritted polypropylene syringe. The resin was then washed with $3 \times 5 \mathrm{~mL} \mathrm{ACN} \mathrm{(1} \mathrm{min} \mathrm{each).} \mathrm{A} \mathrm{solution}$ 
of scaffold 2 ( $82 \mathrm{mg}, 100 \mu \mathrm{mol}$ ), PyBroP (93 mg, $200 \mu \mathrm{mol}, 2.0$ eq.), and DIPEA (26 $\mu \mathrm{L}, 150 \mu \mathrm{mol}, 1.5$ eq.) in ACN $(2.5 \mathrm{~mL})$ was added to the resin. The resulting mixture was stirred at r.t. for $6 \mathrm{~h}$. The resin was washed as described for the determination of the loading of compound $\mathbf{2}$ (vide-infra) and with $3 \times 5 \mathrm{~mL}$ of $\mathrm{MeOH}$ and DCM (1 min each) to afford resin-bound compound $\mathbf{5 s}$.

A small sample of resin-bound compound $\mathbf{5 s}$ ( $c$ a. 10\% of the total amount) was transferred to a fritted glass micro-reactor and dried under reduced pressure. The product was cleaved from the resin with a 5\% TFA/DCM $(v / v)$ solution $(5 \mathrm{~mL})$ for 30 minutes. The resin was subsequently slowly washed with $3 \times 5 \mathrm{~mL}$ of 5\% TFA/DCM. All solutions were combined and evaporated to dryness. It is recommended that the cleavage procedure be repeated until a constant weight of the dried compound is reached. Typically, two TFA cleavage procedures were sufficient to recover all of the material from the resin. Compound $\mathbf{5}$ (12 $\mathrm{mg}$ ) was purified by RP-HPLC (Method A) and lyophilized ( $5.1 \mathrm{mg}$, white powder).

Analytical HPLC: (Method A) $t_{\mathrm{R}}=14.1 \mathrm{~min} .{ }^{1} \mathrm{H}-\mathrm{NMR}\left(500 \mathrm{MHz}\right.$; DMSO- $d 6$; $\left.\mathrm{T}=100{ }^{\circ} \mathrm{C}\right): \delta_{\mathrm{H}} 1.05$ $\left(21 \mathrm{H}, \mathrm{m}, \mathrm{Si}\left(\mathrm{CH}\left(\mathrm{CH}_{3}\right)_{2}\right)_{3}\right), 2.19\left(2 \mathrm{H}, \mathrm{m},-\mathrm{CH}_{2}-\right), 2.28\left(2 \mathrm{H}, \mathrm{br} \mathrm{t},{ }^{3} \mathrm{JHH}_{\mathrm{HH}}=7.5 \mathrm{~Hz},-\mathrm{CH}_{2}-\mathrm{CO}\right), 3.11(1 \mathrm{H}, \mathrm{t}$, $\left.{ }^{4} J_{\mathrm{HH}}=2.4 \mathrm{~Hz},-\mathrm{C} \equiv \mathrm{CH}\right), 4.02\left(2 \mathrm{H}, \mathrm{t},{ }^{3} J_{\mathrm{HF}}=14.5 \mathrm{~Hz},-\mathrm{CH}_{2}-\mathrm{N}\right), 4.18\left(2 \mathrm{H}, \mathrm{d},{ }^{3} J_{\mathrm{HH}}=5.5 \mathrm{~Hz},-\mathrm{CH}_{2}-\mathrm{N}\right)$, $4.24\left(2 \mathrm{H}\right.$, br d, $\left.{ }^{4} J_{\mathrm{HH}}=2.4 \mathrm{~Hz},-\mathrm{CH}_{2}-\mathrm{N}\right), 4.26\left(1 \mathrm{H}, \mathrm{t},{ }^{3} \mathrm{~J}_{\mathrm{HH}}=6.8 \mathrm{~Hz},>\mathrm{CH}-\mathrm{Fmoc}\right), 4.40(2 \mathrm{H}, \mathrm{d}$, ${ }^{3} J_{\mathrm{HH}}=6.8 \mathrm{~Hz},-\mathrm{CH}_{2}-\mathrm{O}$ Fmoc $), 7.30\left(2 \mathrm{H}, \mathrm{t},{ }^{3} J_{\mathrm{HH}}=7.5 \mathrm{~Hz}, 2 \times \mathrm{CH}_{\text {arom }}\right), 7.40\left(2 \mathrm{H}, \mathrm{t},{ }^{3} J_{\mathrm{HH}}=7.5 \mathrm{~Hz}\right.$, $\left.2 \times \mathrm{CH}_{\text {arom }}\right), 7.70\left(2 \mathrm{H}, \mathrm{d},{ }^{3} \mathrm{~J}_{\mathrm{HH}}=7.5 \mathrm{~Hz}, 2 \times \mathrm{CH}_{\text {arom }}\right), 7.84\left(2 \mathrm{H}, \mathrm{d},{ }^{3} \mathrm{~J}_{\mathrm{HH}}=7.5 \mathrm{~Hz}, 2 \times \mathrm{CH}_{\text {arom }}\right), 8.02(1 \mathrm{H}$, $\left.\mathrm{t},{ }^{3} \mathrm{~J}_{\mathrm{HH}}=1.5 \mathrm{~Hz}, \mathrm{CH}_{\text {arom }}\right), 8.04\left(1 \mathrm{H}, \mathrm{t},{ }^{3} \mathrm{~J}_{\mathrm{HH}}=1.5 \mathrm{~Hz}, \mathrm{CH}_{\text {arom }}\right), 8.42\left(1 \mathrm{H}, \mathrm{t},{ }^{3} \mathrm{~J}_{\mathrm{HH}}=1.5 \mathrm{~Hz}, \mathrm{CH}_{\text {arom }}\right), 8.88$ $\left(1 \mathrm{H}\right.$, br t, $\left.{ }^{3} \mathrm{~J}_{\mathrm{HH}}=5.6 \mathrm{~Hz},>\mathrm{NH}\right), 9.08(1 \mathrm{H}, \mathrm{br},>\mathrm{NH}), 10.33(1 \mathrm{H}, \mathrm{br},>\mathrm{NH}) .{ }^{13} \mathrm{C}-\mathrm{NMR}(125.8 \mathrm{MHz}$; DMSO- $\left.\left.d 6 ; \mathrm{T}=100{ }^{\circ} \mathrm{C}\right): \delta \mathrm{C} 10.55\left(3 \mathrm{C}, \mathrm{Si}\left(\mathrm{CH}\left(\mathrm{CH}_{3}\right)_{2}\right) 3\right)\right), 18.09\left(6 \mathrm{C}, \mathrm{Si}\left(\mathrm{CH}\left(\mathrm{CH}_{3}\right)_{2}\right) 3\right), 27.14\left(1 \mathrm{C},-\mathrm{CH}_{2}-\right)$, $29.47\left(1 \mathrm{C},-\mathrm{CH}_{2}-\mathrm{N}\right), 30.04\left(1 \mathrm{C}, \mathrm{t},{ }^{2} J_{\mathrm{CF}}=24.6 \mathrm{~Hz},-\mathrm{CH}_{2}-\right), 38.75\left(1 \mathrm{C},-\mathrm{CH}_{2}-\mathrm{N}\right), 46.56(1 \mathrm{C},>\mathrm{CH}-\mathrm{Fmoc})$, $48.81\left(1 \mathrm{C},-\mathrm{CH}_{2}-\mathrm{N}\right), 66.28\left(1 \mathrm{C},-\mathrm{CH}_{2}-\mathrm{O}\right.$ Fmoc), $75.32(1 \mathrm{C}, \equiv \mathrm{C}-\mathrm{H}), 78.15(1 \mathrm{C}, \equiv \mathrm{C}-), 81.91(1 \mathrm{C}, \equiv \mathrm{C}-)$, $105.15(1 \mathrm{C}, \equiv \mathrm{C}-), 119.67\left(2 \mathrm{C}, 2 \times \mathrm{CH}_{\text {arom }}\right), 123.30\left(1 \mathrm{C}, \mathrm{t},{ }^{1} \mathrm{~J}_{\mathrm{CF}}=244.5 \mathrm{~Hz},>\mathrm{CF}_{2}\right), 124.87(2 \mathrm{C}$, $\left.2 \times \mathrm{CH}_{\text {arom }}\right), 126.74\left(2 \mathrm{C}, 2 \times \mathrm{CH}_{\text {arom }}\right), 127.34\left(2 \mathrm{C}, 2 \times \mathrm{CH}_{\text {arom }}\right), 127.72\left(2 \mathrm{C}, 2 \times \mathrm{CH}_{\text {arom }}\right), 128.09$ (1C, $\left.\mathrm{CH}_{\text {arom }}\right), 133.19$ (1C, $\left.\mathrm{C}_{\text {arom }}\right), 134.90$ (1C, $\left.\mathrm{C}_{\text {arom }}\right), 135.46$ (1C, $\left.\mathrm{C}_{\text {arom }}\right), 140.54$ (2C, $\left.2 \times \mathrm{C}_{\text {arom }}\right), 143.45$ $\left(2 \mathrm{C}, 2 \times \mathrm{C}_{\text {arom }}\right), 155.87(1 \mathrm{C},-\mathrm{CO}-\mathrm{NH}), 164.54$ (1C, -CO-NH), 169.90 (1C, -CO-NH), 172.26 (1C, $\left.-\mathrm{CO}-\mathrm{NH}_{2}\right)\left(-\mathrm{CO}-\mathrm{N}<\right.$ missing). HRMS $(m / z)$ : calcd for $\mathrm{C}_{44} \mathrm{H}_{51} \mathrm{~F}_{2} \mathrm{~N}_{5} \mathrm{NaO}_{6} \mathrm{Si}^{+}[\mathrm{M}+\mathrm{Na}]^{+} 834.34689$, found 834.34718 .

\subsubsection{Compound 6}

The resin $5 \mathbf{s}$ was washed with $3 \times 5 \mathrm{~mL}$ of $\mathrm{MeOH}$ and $t \mathrm{BuOH} /$ water $(1: 1, v / v)(1 \mathrm{~min}$ each). The following solutions were sequentially added to the resin: (i) azide 9 (105 mg, $500 \mu \mathrm{mol}, 5.0$ eq., refer to the Supporting Information for the synthesis) in $1 \mathrm{~mL}$ of $t \mathrm{BuOH} /$ water $(1: 1, v / v)$; (ii) sodium ascorbate (100 $\mu \mathrm{L}$ of a freshly prepared $0.5 \mathrm{M}$ aqueous solution, $50 \mu \mathrm{mol}, 0.5$ eq.) in $1 \mathrm{~mL}$ of $t \mathrm{BuOH} / \mathrm{water}(1: 1$, $v / v)$; and (iii) copper(II) sulfate pentahydrate $(100 \mu \mathrm{L}$ of a freshly prepared $0.1 \mathrm{M}$ aqueous solution, $10 \mu \mathrm{mol}$, 0.1 eq.) in $1 \mathrm{~mL}$ of $t$-BuOH/water $(1: 1, v / v)$. After stirring for $16 \mathrm{~h}$ at r.t., the coupling was complete. The resin was washed with $3 \times 5 \mathrm{~mL}$ of $t \mathrm{BuOH} /$ water $(1: 1, v / v)$, DMF, DCM, MeOH and DCM $(1 \mathrm{~min}$ each) to afford the resin-bound compound $\mathbf{6 s .}$

A small sample of resin-bound compound $\mathbf{6 s}$ ( $c a .10 \%$ of the total amount) was transferred to a fritted glass micro-reactor and dried under reduced pressure. The product was cleaved using the procedure 
described for compound 5 to give crude compound $\mathbf{6}$ ( $c$ a. $18 \mathrm{mg}$ ). Compound $\mathbf{6}$ was purified by RP-HPLC (Method A) and lyophilized (7.3 mg, white powder).

Analytical HPLC: (Method A) $t_{\mathrm{R}}=17.4 \mathrm{~min} .{ }^{1} \mathrm{H}-\mathrm{NMR}\left(500 \mathrm{MHz}\right.$; DMSO- $\left.d_{6} ; \mathrm{T}=100{ }^{\circ} \mathrm{C}\right): \delta_{\mathrm{H}} 1.05$ $\left(21 \mathrm{H}, \mathrm{m}, \mathrm{Si}\left(\mathrm{CH}\left(\mathrm{CH}_{3}\right)_{2}\right)_{3}\right), 2.18\left(2 \mathrm{H}, \mathrm{m},-\mathrm{CH}_{2}-\right), 2.26\left(2 \mathrm{H}, \mathrm{m},-\mathrm{CH}_{2}-\mathrm{CO}\right), 3.93\left(2 \mathrm{H}, \mathrm{t}, J_{\mathrm{HF}}=14.3 \mathrm{~Hz}\right.$, $\left.-\mathrm{CH}_{2}-\mathrm{N}\right), 4.17\left(2 \mathrm{H}, \mathrm{d},{ }^{3} \mathrm{~J}_{\mathrm{HH}}=5.6 \mathrm{~Hz},-\mathrm{CH}_{2}-\mathrm{N}\right), 4.26\left(1 \mathrm{H}, \mathrm{t},{ }^{3} \mathrm{~J}_{\mathrm{HH}}=7.0 \mathrm{~Hz},>\mathrm{CH}-\mathrm{Fmoc}\right), 4.39(2 \mathrm{H}, \mathrm{d}$, ${ }^{3} J_{\mathrm{HH}}=7.0 \mathrm{~Hz},-\mathrm{CH}_{2}-\mathrm{O}$ Fmoc $), 4.70\left(2 \mathrm{H}, \mathrm{s},-\mathrm{CH}_{2}-\mathrm{N}\right), 5.62\left(2 \mathrm{H}, \mathrm{s},-\mathrm{CH}_{2}-\mathrm{N}\right), 7.24\left(1 \mathrm{H}, \mathrm{d},{ }^{3} \mathrm{~J}_{\mathrm{HH}}=7.5 \mathrm{~Hz}\right.$, $\left.\mathrm{CH}_{\text {arom }}\right), 7.29\left(2 \mathrm{H}, \mathrm{t},{ }^{3} \mathrm{~J}_{\mathrm{HH}}=7.5 \mathrm{~Hz}, 2 \times \mathrm{CH}_{\text {arom }}\right), 7.35\left(1 \mathrm{H}, \mathrm{tt},{ }^{3} \mathrm{~J}_{\mathrm{HH}}=7.5 \mathrm{~Hz},{ }^{4} J_{\mathrm{HH}}=1.2 \mathrm{~Hz}, \mathrm{CH}\right.$ arom $), 7.39$ $\left(2 \mathrm{H}, \mathrm{t},{ }^{3} J_{\mathrm{HH}}=7.5 \mathrm{~Hz}, 2 \times \mathrm{CH}_{\text {arom }}\right), 7.44\left(3 \mathrm{H}, \mathrm{t},{ }^{3} \mathrm{~J}_{\mathrm{HH}}=7.5 \mathrm{~Hz}, 3 \times \mathrm{CH}_{\text {arom }}\right), 7.59\left(4 \mathrm{H}, \mathrm{m}, 4 \times \mathrm{CH}_{\text {arom }}\right)$, $7.695\left(2 \mathrm{H}, \mathrm{d},{ }^{3} \mathrm{~J}_{\mathrm{HH}}=7.5 \mathrm{~Hz}, 2 \times \mathrm{CH}_{\text {arom }}\right), 7.84\left(2 \mathrm{H}, \mathrm{d},{ }^{3} \mathrm{~J}_{\mathrm{HH}}=7.5 \mathrm{~Hz}, 2 \times \mathrm{CH}_{\text {arom }}\right), 8.00(1 \mathrm{H}$, br s, CHarom), $8.04\left(1 \mathrm{H}\right.$, br s, $\left.\mathrm{CH}_{\text {arom }}\right), 8.05\left(1 \mathrm{H}\right.$, br s, $\left.\mathrm{CH}_{\text {arom }}\right), 8.40\left(1 \mathrm{H}\right.$, br s, $\left.\mathrm{CH}_{\text {arom }}\right), 8.86\left(1 \mathrm{H}, \mathrm{t},{ }^{3} \mathrm{JHH}_{\mathrm{HH}}=5.6 \mathrm{~Hz},>\mathrm{NH}\right)$, $9.10(1 \mathrm{H}, \mathrm{br},>\mathrm{NH}), 10.32(1 \mathrm{H}, \mathrm{br},>\mathrm{NH}) .{ }^{13} \mathrm{C}-\mathrm{NMR}\left(125.8 \mathrm{MHz}\right.$; DMSO-d6; $\left.\mathrm{T}=100{ }^{\circ} \mathrm{C}\right): \delta \mathrm{C} 10.50(3 \mathrm{C}$, $\left.\left.\mathrm{Si}\left(\mathrm{CH}\left(\mathrm{CH}_{3}\right)_{2}\right)_{3}\right)\right), 18.04\left(6 \mathrm{C}, \mathrm{Si}\left(\mathrm{CH}\left(\mathrm{CH}_{3}\right)_{2}\right)_{3}\right), 27.22\left(1 \mathrm{C},-\mathrm{CH}_{2}-\right), 29.41\left(1 \mathrm{C},-\mathrm{CH}_{2}-\mathrm{N}\right), 30.09(1 \mathrm{C}$, $\left.-\mathrm{CH}_{2}-\right), 43.89\left(1 \mathrm{C},-\mathrm{CH}_{2}-\mathrm{N}\right), 45.51(1 \mathrm{C},>\mathrm{CH}-\mathrm{Fmoc}), 48.56\left(1 \mathrm{C},-\mathrm{CH}_{2}-\mathrm{N}\right), 52.75\left(1 \mathrm{C},-\mathrm{CH}_{2}-\mathrm{N}\right)$,

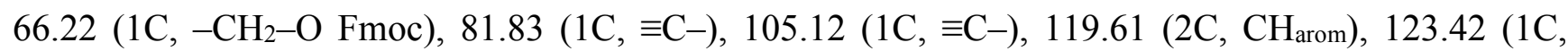
$\left.\mathrm{CH}_{\text {arom}}\right), 124.82$ (2C, $\left.2 \times \mathrm{CH}_{\text {arom }}\right), 126.01$ (1C, $\left.\mathrm{CH}_{\text {arom }}\right), 126.10$ (1C, $\left.\mathrm{CH}_{\text {arom }}\right), 126.34$ (2C, $\left.2 \times \mathrm{CH}_{\text {arom}}\right)$,

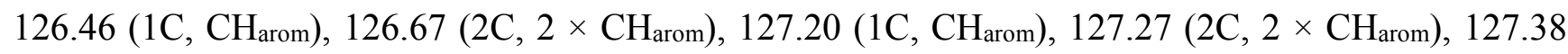

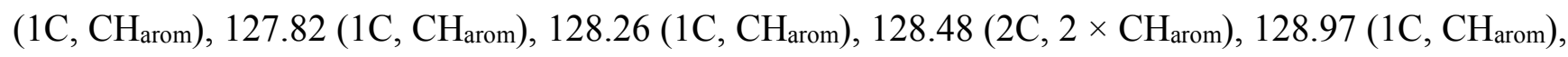
134.76 (1C, $\left.\mathrm{C}_{\text {arom }}\right), 136.01$ (1C, $\left.\mathrm{C}_{\text {arom }}\right), 136.10$ (1C, $\left.\mathrm{C}_{\text {arom }}\right), 139.52$ (1C, $\left.\mathrm{C}_{\text {arom }}\right), 140.49$ (2C, $\left.2 \times \mathrm{C}_{\text {arom }}\right)$, 140.55 (1C, $\left.\mathrm{C}_{\text {arom}}\right), 143.40$ (2C, $\left.2 \times \mathrm{C}_{\text {arom}}\right), 155.82$ (1C, $\left.-\mathrm{CO}-\mathrm{NH}\right), 164.55(1 \mathrm{C},-\mathrm{CO}-\mathrm{NH}), 170.18$ $(1 \mathrm{C},-\mathrm{CO}-\mathrm{NH}), 172.01\left(1 \mathrm{C},-\mathrm{CO}-\mathrm{NH}_{2}\right)(-\mathrm{CO}-\mathrm{N}<$ and two Carom missing). HRMS $(\mathrm{m} / \mathrm{z})$ : calcd for $\mathrm{C}_{57} \mathrm{H}_{62} \mathrm{~F}_{2} \mathrm{~N}_{8} \mathrm{NaO}_{6} \mathrm{Si}^{+}[\mathrm{M}+\mathrm{Na}]^{+} 1043.44219$, found 1043.44263 .

\subsubsection{Compound 7}

(1) Capping: The resin $6 \mathrm{~s}$ was washed with $3 \times 5 \mathrm{~mL}$ of DCM $\left(1 \mathrm{~min}\right.$ each). A cooled solution $\left(0{ }^{\circ} \mathrm{C}\right)$ of $\mathrm{Boc}_{2} \mathrm{O}$ (ca. $220 \mathrm{mg}, c a .1 \mathrm{mmol}$, ca. 10 eq.) and DIPEA (35 $\mu \mathrm{L}, 200 \mu \mathrm{mol}, 2.0$ eq.) in DCM $(4 \mathrm{~mL})$ was added to the resin. The resin was left upside down (to avoid projection) at r.t. for $3 \mathrm{~h}$. The resin was washed with $3 \times 5 \mathrm{~mL}$ of DCM, DMF, DCM and $\mathrm{MeOH}$ ( 1 min each). A Kaiser's test performed on a few grains of resin confirmed complete capping (negative test).

(2) One-step Fmoc-deprotection/hydrazone formation: The Boc-capped resin was washed with $3 \times 5 \mathrm{~mL}$ of DCM and ACN (1 min each). A solution of 3-(trifluoromethyl)benzaldehyde (67 $\mu \mathrm{L}$, $500 \mu \mathrm{mol}, 5.0$ eq.) in $10 \% \mathrm{TEA} / \mathrm{ACN}(v / v, 2.5 \mathrm{~mL}$ ) was added to the resin. The mixture was stirred at r.t. for $16 \mathrm{~h}$. The resin was then washed with $3 \times 5 \mathrm{~mL}$ of ACN, DMF, DCM, MeOH, and DCM (1 min each) to afford resin-bound compound $7 \mathbf{s}$.

A small sample of resin-bound compound $7 \mathbf{s}$ ( $\mathrm{ca}$. 30\% of the total amount) was transferred to a fritted glass micro-reactor and dried under reduced pressure. The product was cleaved using the procedure described for compound 5 to give $24 \mathrm{mg}$ of yellow oil. Compound 7 partially hydrolyzed in the HPLC buffer $(0.1 \% \mathrm{TFA}, \mathrm{pH} \approx 2)$ and was thus purified as follows. First, the low-polarity impurities were extracted from the crude compound 7 with 2 min of sonication in $10 \mathrm{~mL}$ of $\mathrm{Et}_{2} \mathrm{O}$. After centrifugation ( $7200 \mathrm{rpm}, 5 \mathrm{~min}$ at $20^{\circ} \mathrm{C}$ ), the supernatant was discarded. The procedure was repeated once, and the final residue was dried to give $16 \mathrm{mg}$ of a pale-yellow solid. Then, the crude product was passed through 
a C-4 cartridge to remove salts (particularly any remaining copper). Briefly, the crude compound was dissolved in a $30 \%$ aqueous $\mathrm{AcOH}$ solution ( $2 \mathrm{mg} / \mathrm{mL}$ solution) and loaded onto an activated C-4 cartridge (Chromabond C-4, $3 \mathrm{~mL} / 500 \mathrm{mg}$ ). The cartridge was then washed with a $10 \%$ aqueous $\mathrm{AcOH}$ solution $(3 \times 2 \mathrm{~mL})$ and water $(3 \times 2 \mathrm{~mL})$. The product was then eluted with ACN $(5 \times 2 \mathrm{~mL})$ and lyophilized to give 7 (10 mg, white powder). The purity of crude compound 7 was verified by RP-HPLC (Method A, Supplementary Figure S5). This crude compound was directly used for NMR and MS analyses.

Analytical HPLC: (Method A) $t_{\mathrm{R}}=17.7 \mathrm{~min} .{ }^{1} \mathrm{H}-\mathrm{NMR}\left(500 \mathrm{MHz}\right.$; DMSO- $\left.d_{6} ; \mathrm{T}=100{ }^{\circ} \mathrm{C}\right): \delta_{\mathrm{H}} 1.05$ $\left(21 \mathrm{H}, \mathrm{m}, \mathrm{Si}\left(\mathrm{CH}\left(\mathrm{CH}_{3}\right)_{2}\right)_{3}\right), 2.18\left(2 \mathrm{H}, \mathrm{m},-\mathrm{CH}_{2}-\right), 2.26\left(2 \mathrm{H}, \mathrm{m},-\mathrm{CH}_{2}-\mathrm{CO}\right), 3.94\left(2 \mathrm{H}, \mathrm{t}, J_{\mathrm{HF}}=14.2 \mathrm{~Hz}\right.$, $\left.-\mathrm{CH}_{2}-\mathrm{N}\right), 4.18\left(2 \mathrm{H}, \mathrm{d},{ }^{3} \mathrm{~J}_{\mathrm{HH}}=5.6 \mathrm{~Hz},-\mathrm{CH}_{2}-\mathrm{N}\right), 4.72\left(2 \mathrm{H}, \mathrm{s},-\mathrm{CH}_{2}-\mathrm{N}\right), 5.64\left(2 \mathrm{H}, \mathrm{s},-\mathrm{CH}_{2}-\mathrm{N}\right), 7.25(1 \mathrm{H}$, $\left.\mathrm{d},{ }^{3} J_{\mathrm{HH}}=7.5 \mathrm{~Hz}, \mathrm{CH}_{\text {arom }}\right), 7.35\left(1 \mathrm{H}, \mathrm{tt},{ }^{3} J_{\mathrm{HH}}=7.5 \mathrm{~Hz},{ }^{4} J_{\mathrm{HH}}=1.2 \mathrm{~Hz}, \mathrm{CH}_{\text {arom }}\right), 7.44\left(3 \mathrm{H}, \mathrm{t},{ }^{3} J_{\mathrm{HH}}=7.5 \mathrm{~Hz}\right.$, $\left.3 \times \mathrm{CH}_{\text {arom }}\right), 7.59\left(4 \mathrm{H}, \mathrm{m}, 4 \times \mathrm{CH}_{\text {arom }}\right), 7.68\left(1 \mathrm{H}, \mathrm{t},{ }^{3} \mathrm{~J}_{\mathrm{HH}}=7.6 \mathrm{~Hz}, \mathrm{CH}_{\text {arom }}\right), 7.76\left(1 \mathrm{H}, \mathrm{d},{ }^{3} \mathrm{JHH}_{\mathrm{HH}}=7.6 \mathrm{~Hz}\right.$, $\left.\mathrm{CH}_{\text {arom }}\right), 7.99\left(1 \mathrm{H}, \mathrm{d},{ }^{3} \mathrm{~J}_{\mathrm{HH}}=7.6 \mathrm{~Hz}, \mathrm{CH}_{\text {arom }}\right), 8.02\left(2 \mathrm{H}\right.$, br s, $\left.2 \times \mathrm{CH}_{\text {arom }}\right), 8.07\left(1 \mathrm{H}\right.$, br s, $\left.\mathrm{CH}_{\text {arom }}\right), 8.11$ $\left(1 \mathrm{H}\right.$, br s, $\left.\mathrm{CH}_{\text {arom }}\right), 8.45\left(1 \mathrm{H}, \mathrm{t},{ }^{3} \mathrm{~J}_{\mathrm{HH}}=1.6 \mathrm{~Hz}, \mathrm{CH}_{\text {arom }}\right), 8.56(1 \mathrm{H}, \mathrm{s},-\mathrm{N}=\mathrm{CH}), 8.91\left(1 \mathrm{H}, \mathrm{t},{ }^{3} \mathrm{~J}_{\mathrm{HH}}=5.6 \mathrm{~Hz}\right.$, $>\mathrm{NH}), 11.89\left(1 \mathrm{H}\right.$, br s, $>\mathrm{NH}$ ) (one $\mathrm{CH}_{2}-\mathrm{N}$ missing). ${ }^{13} \mathrm{C}-\mathrm{NMR}\left(125.8 \mathrm{MHz}\right.$; DMSO- $\left.d_{6} ; \mathrm{T}=100{ }^{\circ} \mathrm{C}\right): \delta \mathrm{C}$ 10.49 (3C, $\left.\left.\mathrm{Si}\left(\mathrm{CH}\left(\mathrm{CH}_{3}\right)_{2}\right)_{3}\right)\right), 18.03\left(6 \mathrm{C}, \mathrm{Si}\left(\mathrm{CH}_{\left.\left(\mathrm{CH}_{3}\right)_{2}\right)}\right), 27.10\left(1 \mathrm{C},-\mathrm{CH}_{2}-\right), 29.40\left(1 \mathrm{C},-\mathrm{CH}_{2}-\mathrm{N}\right), 30.08\right.$ $\left(1 \mathrm{C},-\mathrm{CH}_{2}-\right), 44.00\left(1 \mathrm{C},-\mathrm{CH}_{2}-\mathrm{N}\right), 52.76\left(1 \mathrm{C},-\mathrm{CH}_{2}-\mathrm{N}\right), 81.82(1 \mathrm{C}, \equiv \mathrm{C}-), 105.11(1 \mathrm{C}, \equiv \mathrm{C}-), 122.82$

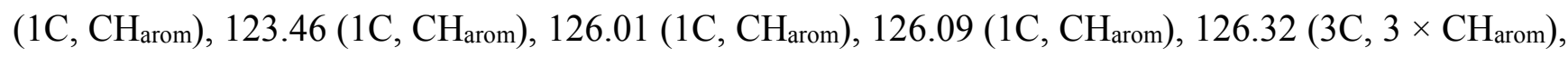
126.43 (1C, $\left.\mathrm{CH}_{\text {arom}}\right), 127.18$ (1C, $\left.\mathrm{CH}_{\text {arom }}\right), 127.74$ (1C, $\left.\mathrm{CH}_{\text {arom }}\right), 128.11$ (1C, $\left.\mathrm{CH}_{\text {arom }}\right), 128.47$ (2C, $\left.2 \times \mathrm{CH}_{\text {arom }}\right), 128.96$ (1C, $\left.\mathrm{CH}_{\text {arom }}\right), 129.57\left(1 \mathrm{C}, \mathrm{CH}_{\text {arom }}\right), 130.56\left(1 \mathrm{C}, \mathrm{CH}_{\text {arom }}\right), 139.50$ (2C, $\left.2 \times \mathrm{C}_{\text {arom }}\right)$, $140.55\left(1 \mathrm{C}, \mathrm{C}_{\text {arom }}\right), 164.56(1 \mathrm{C},-\mathrm{CO}-\mathrm{NH}), 170.20(1 \mathrm{C},-\mathrm{CO}-\mathrm{NH})\left(-\mathrm{CO}-\mathrm{N}<,-\mathrm{CO}-\mathrm{NH}\right.$ and eight $\mathrm{C}_{\text {arom }}$ missing). HRMS $(m / z)$ : calcd for $\mathrm{C}_{50} \mathrm{H}_{56} \mathrm{~F}_{5} \mathrm{~N}_{8} \mathrm{O}_{4} \mathrm{Si}^{+}[\mathrm{M}+\mathrm{H}]^{+}$955.41085, found 955.41159.

\subsubsection{Compound 8 (Low Copper Load)}

(1) TIPS deprotection: The resin $7 \mathbf{s}$ was washed with $3 \times 5 \mathrm{~mL}$ of DMF (1 min each). A solution of TBAF ( $500 \mu \mathrm{L}$ of a $1 \mathrm{M}$ solution in DMF, $500 \mu \mathrm{mol}, 5.0$ eq.) in DMF (2 mL) was then added to the resin. Three treatments at r.t. for $1.5 \mathrm{~h}$ each were necessary to completely remove the TIPS group. Between each treatment, the resin was washed with $3 \times 5 \mathrm{~mL}$ of DMF ( 1 min each). The resin was finally washed with $3 \times 5 \mathrm{~mL}$ of DMF, glacial $\mathrm{AcOH}$, water, $\mathrm{DMF}$, DCM, $\mathrm{MeOH}$, and $\operatorname{DCM}(1$ min each).

(2) Final CuAAC: The resin (terminal-alkyne-bound compound) was washed with $3 \times 5 \mathrm{~mL}$ of $\mathrm{MeOH}$ and $t \mathrm{BuOH} /$ water $(1: 1, v / v)(1 \mathrm{~min}$ each). The following solutions were sequentially added to the resin: (i) 1-azidoheptane 10 (71 mg, $500 \mu \mathrm{mol}, 5.0$ eq., refer to Supplementary Materials for the synthesis) in $1 \mathrm{~mL}$ of $t \mathrm{BuOH} /$ water $(1: 1, v / v)$; (ii) sodium ascorbate $(100 \mu \mathrm{L}$ of a freshly prepared $0.5 \mathrm{M}$ aqueous solution, $50 \mu \mathrm{mol}, 0.5$ eq.) in $1 \mathrm{~mL}$ of $t \mathrm{BuOH} /$ water $(1: 1, v / v)$; and (iii) copper(II) sulfate pentahydrate (100 $\mu \mathrm{L}$ of a freshly prepared $0.1 \mathrm{M}$ aqueous solution, $10 \mu \mathrm{mol}, 0.1 \mathrm{eq}$.) in $1 \mathrm{~mL}$ of $t-\mathrm{BuOH} /$ water $(1: 1, v / v)$. Two couplings of $16 \mathrm{~h}$ and $4 \mathrm{~h}$ at r.t. were necessary for the reaction to reach completion. The resin was washed with $3 \times 5 \mathrm{~mL}$ of $t \mathrm{BuOH} /$ water $(1: 1, v / v)$, DMF, DCM, MeOH and DCM (1 min each) to afford resin-bound compound $\mathbf{8 s .}$

Compound $\mathbf{8}$ was also prepared as described above without partial TFA cleavages to determine the yield of the entire synthesis. The synthesis was performed on a $100 \mu \mathrm{mol}$ scale (using $100 \mu \mathrm{mol}$ of 
scaffold 2 and $200 \mu \mathrm{mol}$ of the Ramage ChemMatrix resin). After the final TFA cleavage, $57 \mathrm{mg}$ of crude compound $\mathbf{8}$ were obtained. The crude compound was extracted twice with $\mathrm{Et}_{2} \mathrm{O}$ and passed through a C-4 cartridge, as explained for compound 7, to afford compound $\mathbf{8}$ ( $23 \mathrm{mg}$, pale yellow solid, overall yield from compound $\mathbf{2}$ of $24 \%$ ). The purity of compound $\mathbf{8}$ was verified by RP-HPLC (Method B, Supplementary Figure S6). Crude compound $\mathbf{8}$ was also analyzed by elemental analysis to check for the presence of residual copper, and ${ }^{19} \mathrm{~F}-\mathrm{NMR}$ was used to quantify the amount of compounds containing the $\mathrm{CF}_{2}$ arm present in the crude product (vide infra).

Analytical HPLC: (Method B) $t_{\mathrm{R}}=22.4 \mathrm{~min}$, purity $=71 \% .{ }^{1} \mathrm{H}-\mathrm{NMR}\left(500 \mathrm{MHz}\right.$; DMSO- $\left.d 6 ; \mathrm{T}=100{ }^{\circ} \mathrm{C}\right)$ : $\delta_{\mathrm{H}} 0.85\left(3 \mathrm{H}, \mathrm{t},{ }^{3} \mathrm{~J}_{\mathrm{HH}}=7.0 \mathrm{~Hz},-\mathrm{CH}_{3}\right), 1.25-1.29\left(8 \mathrm{H}, \mathrm{m}, 4 \times-\mathrm{CH}_{2}-\right), 1.82\left(2 \mathrm{H}, \mathrm{m},-\mathrm{CH}_{2}-\right), 2.18(2 \mathrm{H}, \mathrm{m}$, $\left.-\mathrm{CH}_{2}-\right), 2.26\left(2 \mathrm{H}, \mathrm{m},-\mathrm{CH}_{2}-\mathrm{CO}\right), 3.94\left(2 \mathrm{H}, \mathrm{t}, J_{\mathrm{HF}}=14.2 \mathrm{~Hz},-\mathrm{CH}_{2}-\mathrm{N}\right), 4.30\left(2 \mathrm{H}, \mathrm{t},{ }^{3} \mathrm{JHH}_{\mathrm{HH}}=7.2,-\mathrm{CH}_{2}-\mathrm{N}\right)$, $4.57\left(2 \mathrm{H}, \mathrm{d},{ }^{3} \mathrm{~J}_{\mathrm{HH}}=5.6 \mathrm{~Hz},-\mathrm{CH}_{2}-\mathrm{N}\right), 4.71\left(2 \mathrm{H}, \mathrm{s},-\mathrm{CH}_{2}-\mathrm{N}\right), 5.64\left(2 \mathrm{H}, \mathrm{s},-\mathrm{CH}_{2}-\mathrm{N}\right), 7.25(1 \mathrm{H}, \mathrm{d}$, ${ }^{3} J_{\mathrm{HH}}=7.5 \mathrm{~Hz}, \mathrm{CH}$ arom $), 7.35\left(1 \mathrm{H}, \mathrm{tt},{ }^{3} J_{\mathrm{HH}}=7.4 \mathrm{~Hz},{ }^{4} J_{\mathrm{HH}}=1.2 \mathrm{~Hz}, \mathrm{CH}\right.$ arom $), 7.44\left(3 \mathrm{H}, \mathrm{m}, 3 \times \mathrm{CH}_{\text {arom }}\right)$, $7.60\left(4 \mathrm{H}, \mathrm{m}, 4 \times \mathrm{CH}_{\text {arom }}\right), 7.68\left(1 \mathrm{H}, \mathrm{t},{ }^{3} \mathrm{~J}_{\mathrm{HH}}=7.5 \mathrm{~Hz}, \mathrm{CH}_{\text {arom }}\right), 7.76\left(1 \mathrm{H}, \mathrm{d},{ }^{3} \mathrm{~J}_{\mathrm{HH}}=7.5 \mathrm{~Hz}, \mathrm{CH}_{\text {arom }}\right), 7.895$ $(1 \mathrm{H}, \mathrm{s}, \mathrm{CH}$ arom $), 7.99\left(1 \mathrm{H}, \mathrm{d},{ }^{3} \mathrm{~J}_{\mathrm{HH}}=7.5 \mathrm{~Hz}, \mathrm{CH}_{\text {arom }}\right), 8.025\left(1 \mathrm{H}, \mathrm{s}, \mathrm{CH}_{\text {arom }}\right), 8.03\left(1 \mathrm{H}, \mathrm{s}, \mathrm{CH}_{\text {arom }}\right), 8.10$ $\left(1 \mathrm{H}\right.$, br s, CHarom), $8.11(1 \mathrm{H}$, br s, CHarom $), 8.48\left(1 \mathrm{H}, \mathrm{t},{ }^{4} J_{\mathrm{HH}}=1.6 \mathrm{~Hz}, \mathrm{CH}_{\text {arom }}\right), 8.57(1 \mathrm{H}, \mathrm{s},-\mathrm{N}=\mathrm{CH})$, $8.94\left(1 \mathrm{H}, \mathrm{t},{ }^{3} \mathrm{~J}_{\mathrm{HH}}=5.6 \mathrm{~Hz},>\mathrm{NH}\right), 11.88(1 \mathrm{H}, \mathrm{br} \mathrm{s},>\mathrm{NH}) .{ }^{13} \mathrm{C}-\mathrm{NMR}\left(125.8 \mathrm{MHz}\right.$; DMSO-d 6 ; $\left.\mathrm{T}=100{ }^{\circ} \mathrm{C}\right)$ : $\delta_{\mathrm{C}} 13.23\left(1 \mathrm{C},-\mathrm{CH}_{3}\right), 21.42\left(1 \mathrm{C},-\mathrm{CH}_{2}-\right), 25.50\left(1 \mathrm{C},-\mathrm{CH}_{2}-\right), 27.10\left(1 \mathrm{C},-\mathrm{CH}_{2}-\right), 27.55\left(1 \mathrm{C},-\mathrm{CH}_{2}-\right)$, $29.24\left(2 \mathrm{C}, 2 \times-\mathrm{CH}_{2}-\right), 30.08\left(1 \mathrm{C},-\mathrm{CH}_{2}-\right), 30.59\left(1 \mathrm{C},-\mathrm{CH}_{2}-\right), 34.90\left(1 \mathrm{C},-\mathrm{CH}_{2}-\mathrm{N}\right), 43.90\left(1 \mathrm{C},-\mathrm{CH}_{2}-\right.$ $\mathrm{N}), 48.73\left(1 \mathrm{C},-\mathrm{CH}_{2}-\mathrm{N}\right), 49.06\left(1 \mathrm{C},-\mathrm{CH}_{2}-\mathrm{N}\right), 52.75\left(1 \mathrm{C},-\mathrm{CH}_{2}-\mathrm{N}\right), 122.32\left(1 \mathrm{C}, \mathrm{CH}_{\text {arom }}\right), 122.84(1 \mathrm{C}$, $\mathrm{CH}_{\text {arom }}$ ), 123.50 (1C, $\left.\mathrm{CH}_{\text {arom }}\right), 126.00$ (1C, $\left.\mathrm{CH}_{\text {arom }}\right), 126.08$ (2C, $\left.2 \times \mathrm{CH}_{\text {arom }}\right), 126.32$ (2C, $\left.2 \times \mathrm{CH}_{\text {arom}}\right)$, 126.43 (1C, $\left.\mathrm{CH}_{\text {arom }}\right), 127.18$ (1C, $\left.\mathrm{CH}_{\text {arom }}\right), 128.14$ (1C, $\left.\mathrm{CH}_{\text {arom }}\right), 128.47$ (3C, $\left.3 \times \mathrm{CH}_{\text {arom }}\right), 128.96(1 \mathrm{C}$, $\mathrm{CH}_{\text {arom }}$ ), 129.57 (1C, $\left.\mathrm{CH}_{\text {arom }}\right), 130.57$ (1C, $\left.\mathrm{CH}_{\text {arom }}\right), 133.75$ (1C, $\left.\mathrm{C}_{\text {arom }}\right), 135.31$ (1C, $\left.\mathrm{C}_{\text {arom }}\right), 135.85$ (1C, Carom), 136.09 (1C, $\left.\mathrm{C}_{\text {arom }}\right), 139.51$ (1C, $\left.\mathrm{C}_{\text {arom}}\right), 140.55$ (1C, $\mathrm{C}_{\text {arom }}$ ), 142.31 (1C, $\left.\mathrm{C}_{\text {arom }}\right), 144.25$ (1C, $\left.\mathrm{C}_{\text {arom}}\right)$, $164.77(1 \mathrm{C},-\mathrm{CO}-\mathrm{NH}), 165.79(1 \mathrm{C},-\mathrm{CO}-\mathrm{NH}), 170.24(1 \mathrm{C},-\mathrm{CO}-\mathrm{NH})\left(-\mathrm{CO}-\mathrm{N}<\right.$ and four $\mathrm{C}_{\text {arom }}$ missing). IR $(\mathrm{KBr}) v_{\max }=3433\left(\mathrm{~s}, \mathrm{vbr}, \mathrm{NH}+\mathrm{H}_{2} \mathrm{O}\right), 1664$ (s, br, $\left.\mathrm{C}=\mathrm{O}\right)$. HRMS $(\mathrm{m} / \mathrm{z})$ : calcd for $\mathrm{C}_{48} \mathrm{H}_{50} \mathrm{~F}_{5} \mathrm{~N}_{11} \mathrm{NaO}_{4}{ }^{+}$ $(\mathrm{M}+\mathrm{Na})^{+}$962.38596, found 962.38613. Elemental analysis $(\mathrm{Cu}): 97-103 \mathrm{ppm}$.

In a parallel experiment, we prepared $\mathbf{8}$ by the same methodology as described above, but, before the final cleavage, the resin was treated with gaseous $\mathrm{H}_{2} \mathrm{~S}$ as described below. We obtained $39 \mathrm{mg}$ of crude compound $\mathbf{8}$ (41 $\mu \mathrm{mol}, 41 \%$, if pure) starting from $82 \mathrm{mg}$ of scaffold $\mathbf{2}(100 \mu \mathrm{mol})$.

\subsubsection{Compound $\mathbf{8}$ (High Copper Load)}

The solid-phase synthesis of compound $\mathbf{8}$ was repeated as described above but used a higher load of copper (II) sulfate as well as sodium ascorbate. Thus, for the two CuAAC reactions (to produce resin-bound compounds $\mathbf{6 s}$ and $\mathbf{8 s}$ ), the following protocol was applied. The resin was washed with $3 \times 5 \mathrm{~mL}$ of $\mathrm{MeOH}$ and $t \mathrm{BuOH} /$ water $(1: 1, v / v ; 1 \mathrm{~min}$ each). The following solutions were sequentially added to the resin: (i) azide 9 or $\mathbf{1 0}$ (500 $\mu \mathrm{mol}, 5.0$ eq.) in $1 \mathrm{~mL}$ of $t \mathrm{BuOH} /$ water $(1: 1, v / v)$; (ii) a solution of sodium ascorbate (99 mg, $500 \mu \mathrm{mol}, 5$ eq.) in $1 \mathrm{~mL}$ of $t \mathrm{BuOH} /$ water $(1: 1, v / v)$; and (iii) copper(II) sulfate pentahydrate $(25 \mathrm{mg}, 100 \mu \mathrm{mol}, 1$ eq.) in $1 \mathrm{~mL}$ of $t$ - $\mathrm{BuOH} /$ water $(1: 1, v / v)$. After stirring for $16 \mathrm{~h}$ at r.t., the coupling was complete. The resin was washed with $3 \times 5 \mathrm{~mL}$ of $t \mathrm{BuOH} /$ water $(1: 1, v / v), \mathrm{H}_{2} \mathrm{O}, \mathrm{AcOH}$ (to solubilize copper(I) salts), $\mathrm{H}_{2} \mathrm{O}, \mathrm{DMF}, \mathrm{DCM}, \mathrm{MeOH}$, and DCM (1 min each). The resin was then 
treated with gaseous hydrogen sulfide (vide infra). Before the final cleavage, the resin was washed with $3 \times 5 \mathrm{~mL}$ DCM and dried over $\mathrm{NaOH}$ pellets at 10-100 Pa. The cleavage mixture $(75 \mathrm{mg})$ was extracted twice with $\mathrm{Et}_{2} \mathrm{O}$, passed through a C-4 cartridge as explained for compound 7, and lyophilized to afford final compound 8 (44 mg, pale yellow solid, overall yield from compound 2 of $47 \%$ ). The purity of compound 8 was verified by RP-HPLC (Method B, Figure S7B).

Analytical HPLC: $($ Method B) purity $=64 \%$. Elemental analysis $(\mathrm{Cu}): 15$ ppm.

\subsubsection{Compound 11}

(1) Acylhydrazone formation: The resin-bound compound 5s (100 $\mu \mathrm{mol}$ of scaffold) was capped with Boc groups as described for compound 7. The resin was washed with $3 \times 5 \mathrm{~mL}$ of DCM, DMF, DCM, MeOH, and ACN (1 min each). A solution of 1-butyraldehyde (45 $\mu \mathrm{L}, 500 \mu \mathrm{mol}$, 5.0 eq. $)$ in $10 \% \mathrm{TEA} / \mathrm{ACN}(v / v, 2.5 \mathrm{~mL})$ was added to the resin. The mixture was stirred at r.t. for $16 \mathrm{~h}$. The resin was then washed with $3 \times 5 \mathrm{~mL}$ of ACN ( $1 \mathrm{~min}$ each).

(2) Acylhydrazone reduction: The resin containing the acylhydrazone derivative was washed with $5 \times 5 \mathrm{~mL}$ of dry EtOH. A cooled solution $\left({ }^{\circ} \mathrm{C}\right)$ of sodium borohydride $(38 \mathrm{mg}, 1000 \mu \mathrm{mol}$, 10 eq. $)$ in dry $\mathrm{EtOH}(3 \mathrm{~mL})$ was added to the resin. The syringe was left upside down (to avoid projection) at $5{ }^{\circ} \mathrm{C}$ for $30 \mathrm{~min}$ and at r.t. for $3 \mathrm{~h}$. The resin was then washed with $3 \times 5 \mathrm{~mL}$ of $\mathrm{MeOH}, \mathrm{H}_{2} \mathrm{O}$, DMF, DCM, MeOH, and DCM (1 min each) to give resin-bound compound $11 \mathrm{~s}$.

A sample of resin-bound compound $11 \mathrm{~s} \mathrm{(} \mathrm{ca} .50 \%$ of the total amount) was transferred to a fritted glass micro-reactor and dried under reduced pressure. The product was cleaved using the procedure described for compound 5. The purity of crude compound 11 (23 mg) was verified by RP-HPLC (Method B, Supplementary Figure S8). Compound 11 was purified using RP-HPLC (Method A) and lyophilized (8.9 mg, yield from scaffold $2 \mathrm{ca}$. 30\%, white powder).

Analytical HPLC: (Method B) $t_{\mathrm{R}}=19.8 \mathrm{~min}$, crude purity $=70 \% .{ }^{1} \mathrm{H}-\mathrm{NMR}\left(500 \mathrm{MHz}\right.$; DMSO- $d_{6}$; $\left.\mathrm{T}=100{ }^{\circ} \mathrm{C}\right): \delta_{\mathrm{H}} 0.92\left(3 \mathrm{H}, \mathrm{t},{ }^{3} \mathrm{~J}_{\mathrm{HH}}=7.5 \mathrm{~Hz}, \mathrm{CH}_{3}\right), 1.06\left(21 \mathrm{H}, \mathrm{m}, \mathrm{Si}\left(\mathrm{CH}\left(\mathrm{CH}_{3}\right)_{2}\right)_{3}\right), 1.40\left(2 \mathrm{H}, \mathrm{m},-\mathrm{CH}_{2}-\right)$, $1.51\left(2 \mathrm{H}, \mathrm{m},-\mathrm{CH}_{2}-\right), 2.19\left(2 \mathrm{H}, \mathrm{m},-\mathrm{CH}_{2}-\right), 2.28\left(2 \mathrm{H}, \mathrm{m},-\mathrm{CH}_{2}-\mathrm{CO}\right), 3.15\left(1 \mathrm{H}, \mathrm{t},{ }^{4} \mathrm{~J}_{\mathrm{HH}}=2.4 \mathrm{~Hz}\right.$, $\left.{ }_{-} \mathrm{C} \equiv \mathrm{CH}\right), 4.01\left(2 \mathrm{H}, \mathrm{t},{ }^{3} \mathrm{~J}_{\mathrm{HF}}=14.5 \mathrm{~Hz},-\mathrm{CH}_{2}-\mathrm{N}\right), 4.17\left(2 \mathrm{H}, \mathrm{d},{ }^{3} J_{\mathrm{HH}}=5.6 \mathrm{~Hz},-\mathrm{CH}_{2}-\mathrm{N}\right), 4.23(2 \mathrm{H}$, br d, $\left.{ }^{4} J_{\mathrm{HH}}=2.4 \mathrm{~Hz},-\mathrm{CH}_{2}-\mathrm{N}\right), 7.98\left(1 \mathrm{H}, \mathrm{t},{ }^{3} J_{\mathrm{HH}}=1.5 \mathrm{~Hz}, \mathrm{CH}_{\text {arom }}\right), 8.00\left(1 \mathrm{H}, \mathrm{t},{ }^{3} J_{\mathrm{HH}}=1.5 \mathrm{~Hz}, \mathrm{CH}_{\text {arom }}\right), 8.38$ $\left(1 \mathrm{H}, \mathrm{t},{ }^{3} J_{\mathrm{HH}}=1.5 \mathrm{~Hz}, \mathrm{CH}_{\text {arom }}\right), 8.87\left(1 \mathrm{H}\right.$, br t, $\left.{ }^{3} J_{\mathrm{HH}}=5.6 \mathrm{~Hz},>\mathrm{NH}\right)\left(\right.$ one $-\mathrm{CH}_{2}-\mathrm{N}$ missing). ${ }^{13} \mathrm{C}-\mathrm{NMR}$ $\left.\left(125.8 \mathrm{MHz} ; \mathrm{DMSO}-d_{6} ; \mathrm{T}=100{ }^{\circ} \mathrm{C}\right): \delta_{\mathrm{C}} 10.50\left(3 \mathrm{C}, \mathrm{Si}\left(\mathrm{CH}\left(\mathrm{CH}_{3}\right)_{2}\right)_{3}\right)\right), 13.22\left(1 \mathrm{C},-\mathrm{CH}_{3}\right), 18.04(6 \mathrm{C}$, $\left.\mathrm{Si}\left(\mathrm{CH}\left(\mathrm{CH}_{3}\right)_{2}\right)_{3}\right), 19.31\left(1 \mathrm{C},-\mathrm{CH}_{2}-\right), 27.08\left(1 \mathrm{C},-\mathrm{CH}_{2}-\right), 29.06\left(1 \mathrm{C},-\mathrm{CH}_{2}-\right), 29.39\left(1 \mathrm{C},-\mathrm{CH}_{2}-\mathrm{N}\right), 29.98$ $\left(1 \mathrm{C}, \mathrm{t},{ }^{2} J_{\mathrm{CF}}=24.5 \mathrm{~Hz},-\mathrm{CH}_{2}-\right), 38.50\left(1 \mathrm{C},-\mathrm{CH}_{2}-\mathrm{N}\right), 48.80\left(1 \mathrm{C},-\mathrm{CH}_{2}-\mathrm{N}\right), 50.35\left(1 \mathrm{C},-\mathrm{CH}_{2}-\mathrm{N}\right), 75.14$ $(1 \mathrm{C}, \equiv \mathrm{C}-\mathrm{H}), 78.15(1 \mathrm{C}, \equiv \mathrm{C}-), 81.80(1 \mathrm{C}, \equiv \mathrm{C}-), 105.15(1 \mathrm{C}, \equiv \mathrm{C}-), 123.26\left(1 \mathrm{C}, \mathrm{t},{ }^{1} J_{\mathrm{CF}}=243.8 \mathrm{~Hz},>\mathrm{CF}_{2}\right)$, 127.33 (1C, $\left.\mathrm{CH}_{\text {arom }}\right), 127.43$ (1C, $\left.\mathrm{CH}_{\text {arom }}\right), 127.63$ (1C, $\left.\mathrm{CH}_{\text {arom }}\right), 133.60$ (1C, $\left.\mathrm{C}_{\text {arom }}\right), 134.71$ (1C, C arom), 135.29 (1C, $\left.\mathrm{C}_{\text {arom}}\right), 164.54(1 \mathrm{C},-\mathrm{CO}-\mathrm{NH}), 165.62(1 \mathrm{C},-\mathrm{CO}-\mathrm{NH}), 172.00\left(1 \mathrm{C},-\mathrm{CO}-\mathrm{NH}_{2}\right)(-\mathrm{CO}-\mathrm{N}<$ missing). HRMS (m/z): calcd for $\mathrm{C}_{33} \mathrm{H}_{50} \mathrm{~F}_{2} \mathrm{~N}_{5} \mathrm{O}_{4} \mathrm{Si}^{+}[\mathrm{M}+\mathrm{H}]^{+}$646.35946, found 646.35970. 


\subsubsection{Compound 12}

From resin-bound compound $\mathbf{1 1 s}$, compound 12 was obtained following the procedures described for compounds 6 and 8 (low copper load). After the final TFA cleavage, $20 \mathrm{mg}$ of crude compound 12 were obtained. The crude compound was extracted twice with $\mathrm{Et}_{2} \mathrm{O}$ and passed through a C-4 cartridge, as explained for compound 7. The purity of the crude compound $\mathbf{1 2}$ was verified by RP-HPLC (Method B, Supplementary Figure S9). Crude compound 12 was then purified using RP-HPLC (Method B) and lyophilized to give pure compound $\mathbf{1 2}(3.1 \mathrm{mg}$, white powder).

Analytical HPLC: (Method B) $t_{\mathrm{R}}=17.9 \mathrm{~min} .{ }^{1} \mathrm{H}-\mathrm{NMR}\left(500 \mathrm{MHz}\right.$; DMSO- $\left.d_{6} ; \mathrm{T}=100{ }^{\circ} \mathrm{C}\right): \delta_{\mathrm{H}} 0.86(3 \mathrm{H}$, $\left.\mathrm{t},{ }^{3} J_{\mathrm{HH}}=7.0 \mathrm{~Hz},-\mathrm{CH}_{3}\right), 1.26\left(8 \mathrm{H}, \mathrm{m}, 4 \times-\mathrm{CH}_{2}-\right), 1.81\left(2 \mathrm{H}, \mathrm{m},-\mathrm{CH}_{2}-\right), 2.19\left(2 \mathrm{H}, \mathrm{m},-\mathrm{CH}_{2}-\right), 2.27(2 \mathrm{H}$, $\left.\mathrm{m},-\mathrm{CH}_{2}-\mathrm{CO}\right), 3.91\left(2 \mathrm{H}, \mathrm{t}, J_{\mathrm{HF}}=14.4 \mathrm{~Hz},-\mathrm{CH}_{2}-\mathrm{N}\right), 4.29\left(2 \mathrm{H}, \mathrm{t},{ }^{3} J_{\mathrm{HH}}=7.1,-\mathrm{CH}_{2}-\mathrm{N}\right), 4.56(2 \mathrm{H}, \mathrm{d}$, $\left.{ }^{3} J_{\mathrm{HH}}=5.5 \mathrm{~Hz},-\mathrm{CH}_{2}-\mathrm{N}\right), 4.65\left(2 \mathrm{H}, \mathrm{s},-\mathrm{CH}_{2}-\mathrm{N}\right), 5.64\left(2 \mathrm{H}, \mathrm{s},-\mathrm{CH}_{2}-\mathrm{N}\right), 7.30\left(1 \mathrm{H}, \mathrm{dm},{ }^{3} J_{\mathrm{HH}}=7.5 \mathrm{~Hz}\right.$, $\left.\mathrm{CH}_{\text {arom }}\right), 7.36\left(1 \mathrm{H}, \mathrm{tt},{ }^{3} J_{\mathrm{HH}}=7.5 \mathrm{~Hz},{ }^{4} J_{\mathrm{HH}}=1.2 \mathrm{~Hz}, \mathrm{CH}_{\text {arom }}\right), 7.44\left(3 \mathrm{H}, \mathrm{m}, 3 \times \mathrm{CH}_{\text {arom }}\right), 7.60(4 \mathrm{H}, \mathrm{m}$, $\left.4 \times \mathrm{CH}_{\text {arom }}\right), 7.86\left(1 \mathrm{H}, \mathrm{s}, \mathrm{CH}_{\text {arom }}\right), 8.02\left(1 \mathrm{H}, \mathrm{s}, \mathrm{CH}_{\text {arom }}\right), 8.05\left(1 \mathrm{H}, \mathrm{t},{ }^{4} J_{\mathrm{HH}}=1.6 \mathrm{~Hz}, \mathrm{CH}_{\text {arom }}\right), 8.12(1 \mathrm{H}, \mathrm{t}$, ${ }^{4} J_{\mathrm{HH}}=1.6 \mathrm{~Hz}, \mathrm{CH}$ arom $), 8.50\left(1 \mathrm{H}, \mathrm{t},{ }^{4} J_{\mathrm{HH}}=1.6 \mathrm{~Hz}, \mathrm{CH}_{\text {arom }}\right), 9.02\left(1 \mathrm{H}, \mathrm{t},{ }^{3} J_{\mathrm{HH}}=5.5 \mathrm{~Hz},>\mathrm{NH}\right), 11.89(1 \mathrm{H}$, br s, >NH). ${ }^{13} \mathrm{C}-\mathrm{NMR}\left(125.8 \mathrm{MHz}\right.$; DMSO- $d_{6}$; $\left.\mathrm{T}=100{ }^{\circ} \mathrm{C}\right): \delta_{\mathrm{C}} 13.20\left(1 \mathrm{C},-\mathrm{CH}_{3}\right), 21.42\left(1 \mathrm{C},-\mathrm{CH}_{2}-\right)$, $25.43\left(1 \mathrm{C},-\mathrm{CH}_{2}-\right), 27.11\left(1 \mathrm{C}, \mathrm{t},{ }^{3} J_{\mathrm{CF}}=4.1 \mathrm{~Hz},-\mathrm{CH}_{2}-\right), 27.52\left(1 \mathrm{C},-\mathrm{CH}_{2}-\right), 29.10\left(1 \mathrm{C},-\mathrm{CH}_{2}-\right), 30.06$ $\left(1 \mathrm{C}, \mathrm{t},{ }^{2} J_{\mathrm{CF}}=24.3 \mathrm{~Hz},-\mathrm{CH}_{2}-\right), 30.58\left(1 \mathrm{C},-\mathrm{CH}_{2}-\right), 34.89\left(1 \mathrm{C},-\mathrm{CH}_{2}-\mathrm{N}\right), 44.00\left(1 \mathrm{C},-\mathrm{CH}_{2}-\mathrm{N}\right), 48.62$ $\left(1 \mathrm{C},-\mathrm{CH}_{2}-\mathrm{N}\right), 49.16\left(1 \mathrm{C},-\mathrm{CH}_{2}-\mathrm{N}\right), 52.63\left(1 \mathrm{C},-\mathrm{CH}_{2}-\mathrm{N}\right), 122.73\left(1 \mathrm{C}, \mathrm{CH}_{\text {arom }}\right), 122.80$ (1C, CHarom), 126.07 (1C, $\left.\mathrm{CH}_{\text {arom }}\right), 126.09$ (1C, $\left.\mathrm{CH}_{\text {arom }}\right), 126.43$ (2C, $\left.2 \times \mathrm{CH}_{\text {arom }}\right), 126.58$ (1C, $\left.\mathrm{CH}_{\text {arom }}\right), 127.19$ (1C, $\left.\mathrm{CH}_{\text {arom }}\right), 128.48$ (2C, $\left.2 \times \mathrm{CH}_{\text {arom }}\right), 128.78$ (1C, $\left.\mathrm{CH}_{\text {arom }}\right), 128.90$ (1C, $\left.\mathrm{CH}_{\text {arom }}\right), 129.17\left(1 \mathrm{C}, \mathrm{CH}_{\text {arom }}\right), 129.46$ (1C, CHarom), 131.33 (1C, Carom), 134.89 (1C, Carom), 136.09 (1C, Carom), 136.34 (1C, Carom), 139.53 (1C, Carom), 140.54 (1C, Carom), 141.84 (1C, Carom), 144.73 (1C, Carom), 164.60 (1C, -CO-), 165.71 (1C, $-\mathrm{CO}-), 170.16(1 \mathrm{C},-\mathrm{CO}-), 172.01\left(1 \mathrm{C},-\mathrm{CO}-\mathrm{NH}_{2}\right)$. HRMS $(\mathrm{m} / \mathrm{z})$ : calcd for $\mathrm{C}_{40} \mathrm{H}_{44} \mathrm{~F}_{2} \mathrm{~N}_{9} \mathrm{O}_{5}{ }^{-} 768.34390$ $[\mathrm{M}-\mathrm{H}]^{-}$, found 768.34363; calcd for $\mathrm{C}_{40} \mathrm{H}_{45} \mathrm{~F}_{2} \mathrm{~N}_{9} \mathrm{NaO}_{5}{ }^{+} 792.34039[\mathrm{M}+\mathrm{Na}]^{+}$, found 792.34059 .

\subsubsection{Compound 13}

Compound 13 was synthesized following the procedure described for compound $\mathbf{8}$ (low copper load), replacing the 3-(trifluoromethyl)benzaldehyde in the synthesis of compound 7 with 4-methoxybenzaldehyde (122 $\mu \mathrm{L}, 100 \mu \mathrm{mol}, 10$ eq.) and treating the resin with gaseous hydrogen sulfide after each CuAAC. After the final cleavage, the crude product $\left(56 \mathrm{mg}\right.$ ) was extracted twice with $\mathrm{Et}_{2} \mathrm{O}$, passed through a C-4 cartridge as explained for compound 7, and lyophilized to afford $39 \mathrm{mg}(43 \%)$ of pale-yellow powder. Part of this powder (20 mg) was purified using RP-HPLC (Method A) and lyophilized (5.4 mg, white powder).

Analytical HPLC: $\left(\right.$ Method B) $t_{\mathrm{R}}=20.4 \mathrm{~min} .{ }^{1} \mathrm{H}-\mathrm{NMR}\left(500 \mathrm{MHz}\right.$; DMSO- $\left.d_{6} ; \mathrm{T}=100{ }^{\circ} \mathrm{C}\right): \delta_{\mathrm{H}} 0.85(3 \mathrm{H}$, $\left.\mathrm{t},{ }^{3} \mathrm{JHH}_{\mathrm{HH}}=7.5 \mathrm{~Hz}, \mathrm{CH}_{3}\right), 1.24-1.29\left(8 \mathrm{H}, \mathrm{m}, 4 \times-\mathrm{CH}_{2}-\right), 1.82\left(2 \mathrm{H}, \mathrm{m},-\mathrm{CH}_{2}-\right), 2.19\left(2 \mathrm{H}, \mathrm{m},-\mathrm{CH}_{2}-\right), 2.26$ $\left(2 \mathrm{H}, \mathrm{m},-\mathrm{CH}_{2}-\right), 3.83\left(3 \mathrm{H}, \mathrm{s},-\mathrm{OCH}_{3}\right), 3.94\left(2 \mathrm{H}, \mathrm{brt},{ }^{3} \mathrm{~J}_{\mathrm{HF}}=14.2 \mathrm{~Hz}, \mathrm{~N}-\mathrm{CH}_{2}-\right), 4.30\left(2 \mathrm{H}, \mathrm{t},{ }^{3} \mathrm{~J}_{\mathrm{HH}}=7.1 \mathrm{~Hz}\right.$, $\left.\mathrm{N}-\mathrm{CH}_{2}-\right), 4.57\left(2 \mathrm{H}, \mathrm{d},{ }^{3} \mathrm{~J}_{\mathrm{HH}}=5.6 \mathrm{~Hz}, \mathrm{~N}-\mathrm{CH}_{2}-\right), 4.70(2 \mathrm{H}$, br s, N-CH $2-), 5.63\left(2 \mathrm{H}, \mathrm{s}, \mathrm{N}-\mathrm{CH}_{2}-\right), 7.00$ $\left(2 \mathrm{H}\right.$, br d, $\left.{ }^{3} J_{\mathrm{HH}}=8.5 \mathrm{~Hz}, 2 \times \mathrm{CH}_{\text {arom }}\right), 7.25\left(2 \mathrm{H}\right.$, br d, $\left.{ }^{3} J_{\mathrm{HH}}=7.5 \mathrm{~Hz}, 2 \times \mathrm{CH}_{\text {arom }}\right), 7.36\left(1 \mathrm{H}, \mathrm{m}, \mathrm{CH}_{\text {arom }}\right)$, $7.45\left(3 \mathrm{H}, \mathrm{m}, 3 \times \mathrm{CH}_{\text {arom }}\right), 7.60\left(3 \mathrm{H}, \mathrm{m}, 3 \times \mathrm{CH}_{\text {arom }}\right), 7.65\left(2 \mathrm{H}, \mathrm{br} \mathrm{d},{ }^{3} J_{\mathrm{HH}}=8.5 \mathrm{~Hz}, 2 \times \mathrm{CH}_{\text {arom }}\right), 7.89$ $\left(1 \mathrm{H}, \mathrm{s}, \mathrm{CH}_{\text {arom }}\right), 8.03\left(1 \mathrm{H}, \mathrm{m}, \mathrm{CH}_{\text {arom }}\right), 8.08\left(2 \mathrm{H}, \mathrm{br}, 2 \times \mathrm{CH}_{\text {arom }}\right), 8.46\left(1 \mathrm{H}, \mathrm{br}, \mathrm{CH}_{\text {arom }}\right), 8.93(1 \mathrm{H}, \mathrm{br} \mathrm{d}$, 
$\left.{ }^{3} \mathrm{JHH}_{\mathrm{HH}}=5.6 \mathrm{~Hz}, \mathrm{NH}\right)(-\mathrm{N}=\mathrm{CH}$ missing $) .{ }^{13} \mathrm{C}-\mathrm{NMR}\left(125.8 \mathrm{MHz} ; \mathrm{DMSO}-d_{6} ; \mathrm{T}=100{ }^{\circ} \mathrm{C}\right): \delta \mathrm{C} 13.25(1 \mathrm{C}$, $\left.\mathrm{CH}_{3}\right), 21.45\left(1 \mathrm{C},-\mathrm{CH}_{2}-\right), 25.52\left(1 \mathrm{C},-\mathrm{CH}_{2}-\right), 27.14\left(1 \mathrm{C},-\mathrm{CH}_{2}-\right), 27.57\left(1 \mathrm{C},-\mathrm{CH}_{2}-\right), 29.27\left(1 \mathrm{C},-\mathrm{CH}_{2}-\right)$, $30.11\left(1 \mathrm{C},-\mathrm{CH}_{2}-\right), 30.62\left(1 \mathrm{C},-\mathrm{CH}_{2}-\right), 34.93\left(1 \mathrm{C},-\mathrm{CH}_{2}-\mathrm{N}\right), 43.94\left(1 \mathrm{C},-\mathrm{CH}_{2}-\mathrm{N}\right), 48.66\left(1 \mathrm{C},-\mathrm{CH}_{2}-\mathrm{N}\right)$, $49.08\left(1 \mathrm{C},-\mathrm{CH}_{2}-\mathrm{N}\right), 52.77\left(1 \mathrm{C},-\mathrm{CH}_{2}-\mathrm{N}\right), 55.14\left(1 \mathrm{C}, \mathrm{OCH}_{3}\right), 114.23\left(2 \mathrm{C}, 2 \times \mathrm{CH}_{\text {arom}}\right), 122.33(1 \mathrm{C}$,

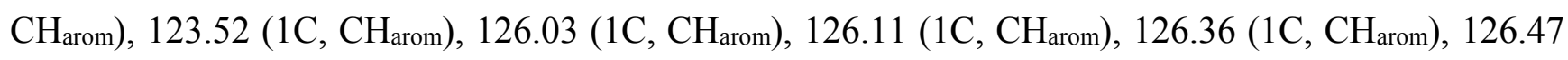
(1C, $\left.\mathrm{CH}_{\text {arom }}\right), 127.22$ (1C, $\left.\mathrm{CH}_{\text {arom}}\right), 127.94$ (1C, $\left.\mathrm{CH}_{\text {arom}}\right), 128.42\left(2 \mathrm{C}, 2 \times \mathrm{CH}_{\text {arom}}\right), 128.51$ (2C, $\left.2 \times \mathrm{CH}_{\text {arom }}\right), 128.99$ (3C, $\left.3 \times \mathrm{CH}_{\text {arom }}\right), 134.13$ (1C, $\left.\mathrm{C}_{\text {arom }}\right), 134.74$ (1C, $\left.\mathrm{C}_{\text {arom }}\right), 135.79$ (1C, $\left.\mathrm{C}_{\text {arom }}\right), 136.14$ (1C, Carom), 139.52 (1C, Carom), 140.57 (1C, Carom), 142.26 (1C, $\left.C_{\text {arom }}\right), 144.30$ (1C, Carom), 160.89 (1C, $\left.\mathrm{C}_{\text {arom }}\right), 164.86(1 \mathrm{C},-\mathrm{CO}-\mathrm{NH})\left(-\mathrm{CO}-\mathrm{N}<,-\mathrm{CO}-\mathrm{NH}_{2},-\mathrm{CO}-\mathrm{NH}\right.$ and three $\mathrm{C}_{\text {arom }}$ missing). HRMS $(\mathrm{m} / z)$ : calcd for $\mathrm{C}_{48} \mathrm{H}_{53} \mathrm{~F}_{2} \mathrm{~N}_{11} \mathrm{NaO}_{5}{ }^{+} 924.40914[\mathrm{M}+\mathrm{Na}]^{+}$, found 924.40973 .

\subsection{Hydrogen Sulfide Treatment}

The resin was washed with $3 \times 5 \mathrm{~mL}$ of ACN and suspended in $5 \mathrm{~mL}$ of ACN. Gaseous hydrogen sulfide was aspirated through the syringe, and the syringe was left for $30 \mathrm{~min}$ (a dark coloration appears). The resin was then washed with $3 \times 5 \mathrm{~mL}$ of ACN and DCM.

CAUTION: hydrogen sulfide is a toxic gas that should be only manipulated under a fume hood.

\subsection{Determination of the Loading of $\mathbf{4}$ on Resin}

(1) Construction of the curve: scaffold 2 (16.5 mg, $20.3 \mu \mathrm{mol})$ was dissolved in 40\% DCM/ACN $(v / v)$. This mother solution was diluted $2,4,8,16$, and 100 times to obtain five solutions at different concentrations. The absorbance of these solutions at $301 \mathrm{~nm}$ (absorption of the Fmoc group) was determined. The experiment was duplicated, and the following equation-linking the absorbance and concentration of scaffold 2-was obtained: $A=4.34 c+0.0828\left(R^{2}=0.999\right)$, where $\mathrm{A}$ is the absorbance and $\mathrm{c}$ the concentration in $\mu \mathrm{M}$.

(2) Loading determination: Scaffold 2 (82 mg, $100 \mu \mathrm{mol})$ was loaded onto Ramage ChemMatrix ${ }^{\circledR}$ resin $(667 \mathrm{mg}, 200 \mu \mathrm{mol})$ following the protocol described for compound $\mathbf{5}$. After the reaction, the resin was washed with $3 \times 5 \mathrm{~mL}$ of ACN, $3 \times 5 \mathrm{~mL}$ of DCM, $5 \mathrm{~mL}$ of $\mathrm{ACN}$, and $5 \mathrm{~mL}$ of DCM (1 min each). The washing solutions were transferred to a $50 \mathrm{~mL}$ volumetric flask filled with ACN. The absorbance of this solution at $301 \mathrm{~nm}$ was measured (blank $=40 \% \mathrm{DCM} / \mathrm{ACN}$ ). The quantity of compound $\mathbf{2}$ present in the solution (unreacted compound) was determined using the equation $\mathrm{A}=4.34 \mathrm{c}+0.0828$. For instance, an absorbance of 1.67 corresponded to $18.3 \mu \mathrm{mol}$ of scaffold 2. Thus, $81.7 \mu \mathrm{mol}(100-18.3 \mu \mathrm{mol})$ of compound should be attached to the resin, which corresponds to a loading of $40.8 \%(=(81.7 / 200) \times 100)$.

\section{5. ${ }^{19}$ F-NMR Quantitative Analysis}

The ${ }^{19} \mathrm{~F}-\mathrm{NMR}$ analysis was carried out as previously described [4]. Briefly, a precisely weighed sample of dry compound $\mathbf{8}\left(4.8 \mathrm{mg}, 5.1 \mu \mathrm{mol}\right.$ if pure) was transferred to a NMR tube with DMSO- $d_{6}(0.4 \mathrm{~mL})$. A known amount of commercial ethyl bromodifluoroacetate $(100 \mu \mathrm{L}$ of a $0.1 \mathrm{M}$ solution in DMSO- $d 6)$ the internal standard - was added to the NMR tube. Three ${ }^{19} \mathrm{~F}$ signals were present for compound $\mathbf{8}$ (refer to the ${ }^{19} \mathrm{~F}-\mathrm{NMR}$ spectrum in Supplementary Figure $\mathrm{S} 11$ ): one for the $\mathrm{CF}_{3}$ group and two for the $\mathrm{CF}_{2}$ group, 
which result from the slow inter-conversion of conformers.[4] The integration over the two $\mathrm{CF}_{2}{ }^{19} \mathrm{~F}$ signals, and the comparison to the standard signal, showed a content of fluorine-containing compounds in the sample of $4.8 \mu \mathrm{mol}$. These data show that our fluorine label can reliably quantify the content of fluorine-containing scaffold-8-related compound(s) by ${ }^{19}$ F-NMR.

\section{Conclusions}

We developed a new variant of a trifunctional scaffold designed for the solid-phase synthesis of combinatorial libraries. The scaffold $\mathbf{2}$ can be substituted by two different azides and an aromatic aldehyde, which extends its scope compared to our first trialkyne-based scaffold $\mathbf{1}$. The derivatization of scaffold 2 with aromatic aldehydes leads, in general, to the desired compounds in sufficient purity for direct biological evaluations. However, electron-rich aromatic hydrazones can be problematic because of their relative instability. Aliphatic aldehydes cannot be used; the resulting aliphatic acylhydrazone is hydrolyzed during cleavage from the resin, and, if reduced to the corresponding aliphatic hydrazide, hydrolysis occurs during the subsequent CuAAC.

We established a one-pot Fmoc-deprotection/acylhydrazone formation protocol that is effective with aromatic aldehydes as well as with aliphatic aldehydes. This methodology is based on the use of TEA as the base for the Fmoc-deprotection. Because Fmoc-hydrazine is easily introduced onto carboxylic acids (through simple amide coupling), this methodology could be of broad use. We solved the problem of the accumulation of interfering copper ions in the resin: treatment of the resin with gaseous hydrogen sulfide traps the copper ions as inert copper sulfide. This protocol is easy to implement and permits the use of one equivalent of copper during the CuAACs.

Overall, scaffold $\mathbf{2}$ offers a new chemical space for the development of bioactive compounds, and the information provided here will be helpful to chemists working on multifunctional scaffolds or performing solid-phase CuAACs.

\section{Supplementary Materials}

Experimental for azido compounds $\mathbf{9}$ and $\mathbf{1 0}$ (Scheme S1), study of the copper-promoted hydrolysis of acylhydrazide in solution (Figure S1), study of the acylhydrazone formation in the presence of copper(II) ions (Figure S2 and Table S1), study of the hydrolysis of $\mathbf{1 3}$ in the presence of copper(II) ions (Table S2, Scheme S5), discussion of the step order (Scheme S6 and Figure S3), RP-HPLC chromatograms for compounds 2, 7, 8 (low and high copper load), 11, 12, and 13 (Figures S4-S10), and ${ }^{19} \mathrm{~F}-\mathrm{NMR}$ spectra for the quantitative analysis of compound $\mathbf{8}$ (Figures S11). Supplementary materials can be accessed at: http://www.mdpi.com/1420-3049/20/10/19310/s1.

\section{Acknowledgments}

This work was supported by the Grant Agency of the Czech Republic (grant 14-17305S, to J.J.), by the Academy of Sciences of the Czech Republic (Research Project RVO: 6138963, support to the Institute of Organic Chemistry and Biochemistry and postdoctoral stipend to B.F.), by the Institute of Organic Chemistry and Biochemistry (IOCB) and the Czech Academy of Sciences (postdoctoral stipend to B.F.) and partly also by the Medical Research Council (Grant MR/K000179/1, to J.J.). 


\section{Author Contributions}

J.J. and B.F. conceived and designed the experiments; B.F., J.P., M.B. and V.V. performed the experiments; B.F. and M.B. analyzed the data; B.F., J.J. and V.V. wrote the paper.

\section{Conflicts of Interest}

The authors declare no conflict of interest.

\section{References}

1. Beal, D.M.; Jones, L.H. Molecular scaffolds using multiple orthogonal conjugations: Applications in chemical biology and drug discovery. Angew. Chem. Int. Ed. 2012, 51, 6320-6326.

2. Iha, R.K.; Wooley, K.L.; Nyström, A.M.; Burke, D.J.; Kade, M.J.; Hawker, C.J. Applications of orthogonal "click" chemistries in the synthesis of functional soft materials. Chem. Rev. 2009, 109, 5620-5686.

3. Chamorro, C.; Kruijtzer, J.A.; Farsaraki, M.; Balzarini, J.; Liskamp, R.M. A general approach for the non-stop solid phase synthesis of tac-scaffolded loops towards protein mimics containing discontinuous epitopes. Chem. Commun. 2009, 7, 821-823.

4. Vaněk, V.; Pícha, J.; Fabre, B.; Buděšínský, M.; Lepšík, M.; Jiráček, J. The development of a versatile trifunctional scaffold for biological applications. Eur. J. Org. Chem. 2015, 17, 3689-3701.

5. Opatz, T.; Liskamp, R.M.J. Synthesis and screening of libraries of synthetic tripodal receptor molecules with three different amino acid or peptide arms: Identification of iron binders. J. Comb. Chem. 2002, 4, 275-284.

6. Valverde, I.E.; Delmas, A.F.; Aucagne, V. Click à la carte: Robust semi-orthogonal alkyne protecting groups for multiple successive azide/alkyne cycloadditions. Tetrahedron 2009, 65, 7597-7602.

7. Nowick, J.S.; Chung, D.M.; Maitra, K.; Maitra, S.; Stigers, K.D.; Sun, Y. An unnatural amino acid that mimics a tripeptide $\beta$-strand and forms $\beta$-sheetlike hydrogen-bonded dimers. J. Am. Chem. Soc. 2000, 122, 7654-7661.

8. Greene, T.W.; Wuts, P.G. Protective Groups in Organic Synthesis, 3rd ed.; John Wiley \& Sons: Hoboken, NJ, USA, 1998.

9. García-Martín, F.; Quintanar-Audelo, M.; García-Ramos, Y.; Cruz, L.J.; Gravel, C.; Furic, R.; Côté, S.; Tulla-Puche, J.; Albericio, F. Chemmatrix, a poly (ethylene glycol)-based support for the solid-phase synthesis of complex peptides. J. Comb. Chem. 2006, 8, 213-220.

10. García-Martín, Y.; Paradís-Bas, M.; Tulla-Puche, J.; Albericio, F. Chemmatrix ${ }^{\circledR}$ for complex peptides and combinatorial chemistry. J. Pept. Sci. 2010, 16, 675-678.

11. Ramage, R.; Irving, S.; McInnes, C. Design of a versatile linker for solid phase peptide synthesis: Synthesis of C-terminal primary/seconary amides and hydrazides. Tetrahedron Lett. 1993, 34, 6599-6602.

12. Ueki, M.; Amemiya, M. Removal of 9-fluorenylmethyloxycarbonyl (fmoc) group with tetrabutylammonium fluoride. Tetrahedron Lett. 1987, 28, 6617-6620.

13. Isaad, A.L.; Papini, A.M.; Chorev, M.; Rovero, P. Side chain-to-side chain cyclization by click reaction. J. Pept. Sci. 2009, 15, 451-454. 
14. Tornoe, C.W.; Christensen, C.; Meldal, M. Peptidotriazoles on solid phase: [1,2,3]-triazoles by regiospecific copper(I)-catalyzed 1,3-dipolar cycloadditions of terminal alkynes to azides. J. Org. Chem. 2002, 67, 3057-3064.

15. Rostovtsev, V.V.; Green, L.G.; Fokin, V.V.; Sharpless, K.B. A stepwise huisgen cycloaddition process: Copper(I)-catalyzed regioselective "ligation" of azides and terminal alkynes. Angew. Chem. Int. Ed. 2002, 41, 2596-2599.

16. Montoya, L.A.; Pluth, M.D. Selective turn-on fluorescent probes for imaging hydrogen sulfide in living cells. Chem. Commun. 2012, 48, 4767-4769.

17. Liu, C.R.; Pan, J.; Li, S.; Zhao, Y.; Wu, L.Y.; Berkman, C.E.; Whorton, A.R.; Xian, M. Capture and visualization of hydrogen sulfide by a fluorescent probe. Angew. Chem. Int. Ed. 2011, 50, 10327-10329.

18. Rao, H.S.P.; Siva, P. Facile reduction of azides with sodium-borohydride copper(II) sulfate system. Synth. Commun. 1994, 24, 549-555.

19. Tian, M.-Z.; Hu, M.-M.; Fan, J.-L.; Peng, X.-J.; Wang, J.-Y.; Sun, S.-G.; Zhang, R. Rhodamine-based "turn-on" fluorescent probe for $\mathrm{Cu}(\mathrm{II})$ and its fluorescence imaging in living cells. Bioorg. Med. Chem. Lett. 2013, 23, 2916-2919.

20. Huang, C.-H.; Stone, A.T. Transformation of the plant growth regulator daminozide (alar) and structurally related compounds with cuii ions: Oxidation versus hydrolysis. Environ. Sci. Technol. 2003, 37, 1829-1837.

21. Kumar, M.; Kumar, N.; Bhalla, V.; Sharma, P.R.; Kaur, T. Highly selective fluorescence turn-on chemodosimeter based on rhodamine for nanomolar detection of copper ions. Org. Lett. 2012, 14, 406-409.

22. Fu, F.L.; Wang, Q. Removal of heavy metal ions from wastewaters: A review. J. Environ. Manage 2011, 92, 407-418.

23. Bradley, D.; Williams, G.; Lawton, M. Drying of organic solvents: Quantitative evaluation of the efficiency of several desiccants. J. Org. Chem. 2010, 75, 8351-8354.

Sample Availability: Samples of the compounds 2, 8, 11 and $\mathbf{1 3}$ are available from the authors.

(C) 2015 by the authors; licensee MDPI, Basel, Switzerland. This article is an open access article distributed under the terms and conditions of the Creative Commons Attribution license (http://creativecommons.org/licenses/by/4.0/). 